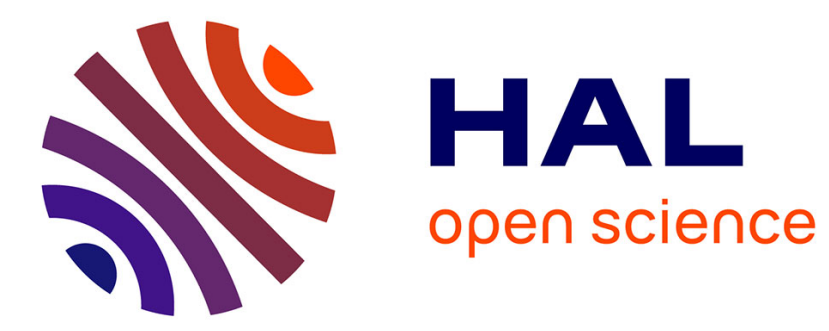

\title{
A Solar Signature in Many Climate Indices
}

\author{
Jean-Louis Le Mouël, Fernand Lopes, Vincent Courtillot
}

\section{To cite this version:}

Jean-Louis Le Mouël, Fernand Lopes, Vincent Courtillot. A Solar Signature in Many Climate Indices. Journal of Geophysical Research: Atmospheres, 2019, 124, pp.2600-2619. 10.1029/2018JD028939 . insu-03586654

\section{HAL Id: insu-03586654 https://hal-insu.archives-ouvertes.fr/insu-03586654}

Submitted on 24 Feb 2022

HAL is a multi-disciplinary open access archive for the deposit and dissemination of scientific research documents, whether they are published or not. The documents may come from teaching and research institutions in France or abroad, or from public or private research centers.
L'archive ouverte pluridisciplinaire HAL, est destinée au dépôt et à la diffusion de documents scientifiques de niveau recherche, publiés ou non, émanant des établissements d'enseignement et de recherche français ou étrangers, des laboratoires publics ou privés. 


\section{JGR Atmospheres}

\author{
RESEARCH ARTICLE \\ 10.1029/2018JD028939 \\ Key Points: \\ - Analysis of long series of solar and \\ terrestrial indices yields many \\ common components \\ - Singular Spectrum analysis is very \\ efficient at extracting meaningful \\ components; we identify common \\ periods of 22 and 11 years, among \\ others \\ - These observations suggest a \\ significant influence of solar \\ variability in driving the variability \\ of many climate indices
}

Correspondence to:

V. Courtillot,

courtil@ipgp.fr

Citation:

Le Mouël, J.-L., Lopes, F., \& Courtillot, V. (2019). A solar signature in many

climate indices. Journal of Geophysical Research: Atmospheres, 124, 2600-2619. https://doi.org/10.1029/2018JD028939

Received 2 MAY 2018

Accepted 10 FEB 2019

Accepted article online 22 FEB 2019

Published online 12 MAR 2019

Author Contributions:

Conceptualization: Jean-Louis Le

Mouël, Fernand Lopes, Vincent

Courtillot

Formal analysis: Jean-Louis Le

Mouël, Fernand Lopes

Investigation: Jean-Louis Le Mouël,

Fernand Lopes, Vincent Courtillot

Methodology: Jean-Louis Le Mouël,

Fernand Lopes

Supervision: Jean-Louis Le Mouël,

Fernand Lopes

Validation: Jean-Louis Le Mouël,

Vincent Courtillot

Writing - original draft: Jean-Louis

Le Mouël, Fernand Lopes

Writing - review \& editing: Jean-

Louis Le Mouël, Fernand Lopes,

Vincent Courtillot

\section{A Solar Signature in Many Climate Indices}

\author{
Jean-Louis Le Mouël ${ }^{1}$, Fernand Lopes ${ }^{1}$, and Vincent Courtillot ${ }^{1}$ iD \\ ${ }^{1}$ Geomagnetism and Paleomagnetism, Institut de Physique du Globe de Paris, Paris, France
}

\begin{abstract}
We first apply singular spectrum analysis (SSA) to the international sunspot number (1849-2015) and the count of polar faculae (1906-2006). The SSA method finds 22-, 11-, and 5.5-year components as the first eigenvectors of these solar activity proxies. We next apply SSA to the 10 Madden-Julian oscillation (MJO; 1978-2016) indices. The first, most intense component SSA finds in all MJO indices has either a period of 5.5 or 11 years. The longer-term modulation of amplitude is on the order of one third of the total variation. The 5.5-year SSA component 1 of most MJO indices moreover follows the decreasing amplitude of solar cycles. We then apply SSA to climate indices Pacific Decadal Oscillation, El Nino Southern Oscillation precipitation index, Arctic Oscillation, Atlantic Multidecadal oscillation, Tropical Southern Atlantic oscillation, Western Hemisphere Warm Pool, and Brazil and Sahel rainfalls. We find that the first SSA eigenvectors are all combinations of rather pure 11, 5.5, and 3.6-year pseudo-cycles. The 5.5-year component is frequently observed and is particularly important and sharp in the series in which it appears. All these periods have long been attributed to solar activity, and this by itself argues for the existence of a strong link between solar activity and climate. The mechanisms of coupling must be complex and probably nonlinear but they remain to be fully understood (UV radiation, solar wind, and galactic cosmic rays being the most promising candidates). We propose as a first step a Kuramoto model of nonlinear coupling that generates phase variations compatible with the observed ones.
\end{abstract}

\section{Introduction}

Singular spectrum analysis (SSA) is a relatively recent and powerful method of spectral analysis that works well with short and noisy time series. It is due to Broomhead and King (1986a, 1986b) and Broomhead et al. (1987). Useful accounts and applications to climatic time series are given by Vautard and Ghil (1989) and Vautard et al. (1992); see also the monograph of Golyandina et al. (2001) and the review by Ghil et al. (2002). Many geophysical time series are "short" in a numerical sense: their length is not very much longer than some of the periodicities that they might contain; moreover, they actually often contain pseudoperiodicities that fluctuate in both amplitude and pseudo-period. SSA provides at the same time a noise reduction technique, a detrending algorithm, and a way to identify oscillatory components (Vautard et al., 1992). SSA yields reconstructed series, in which a small number of components contain a significant amount of the signal variance. SSA does not use prior knowledge of the underlying physics of nonlinear dynamical systems. As recalled by Vautard et al. (1992), it is superior over classical spectral methods, such as Fourier analysis, in that the eigen-elements it is based on are data adaptive.

SSA has been applied to the irregular El Nino Southern Oscillation precipitation index (ENSO) phenomenon, to global-surface temperature, to geopotential height data (some references in Vautard et al., 1992), and to a number of indicators of climate variability (references in Ghil et al.'s, 2002, review). It had not been applied to direct solar observations, such as sunspots, until Le Mouël et al. (2017) identified Gleissberg cycles in three century-long series of sunspot numbers. Usoskin et al. (2016) applied it to cosmogenic isotopes, which provide the only quantitative proxy for analyzing the longer-term solar variability. Lopes et al. (2017) isolated clear, almost periodic 11- and 5.5-year variations in a century-long series of Earth's mantle rotation pole positions. These recent studies all confirm that SSA allows one to identify and extract pseudo-periodic (modulated) oscillations valid for the sampling interval rather than a simple spectral line.

In this paper, we apply the method to a number of solar and climate indices and isolate in all of them a small number of pseudo-periodic components that carry a significant fraction of the signal. They are suggestive of nonlinear couplings of dynamical systems, pointing to interesting avenues of research. These observations are straightforward (as will be seen in Figures $12 \mathrm{~b}$ and 13b), although we have certainly not yet understood the full physical mechanisms that generate them.
(C)2019. American Geophysical Union. All Rights Reserved. 
We start with the sunspot number (ISSN; a proxy for the evolution of solar activity) and the Madden-Julian oscillation (MJO; an important climate phenomenon). Jones and Carvalho (2006) have studied changes in the activity of the MJO during 1958-2004. They find that the MJO has undergone changes in its long period trends and that its periods of high activity are separated by 18.5 years. Blanter et al. (2012) studied aspects of the evolution of the spectral characteristics of MJO over several decades. The typical lifetime of MJO events is in the range 45-50 days; this is also where Fourier spectra of MJO indices reach their maximum amplitude. These spectra display no simple, sharp spectral lines, nor do they reveal sharp periodicities. Yet, using the wave packet technique, Shnirman et al. $(2009,2010)$ and Blanter et al. (2012) were able to identify a solar signature in the spectral characteristics of MJO: there is a good correlation between the mean period of the MJO wave packet and several solar indices, such as ISSN (sunspot number), GCR (galactic cosmic ray index), and MgII (Magnesium core-to-wing ratio index), over three 11-year cycles from 1980 to 2010. Physical mechanisms responsible for the MJO are not yet fully understood: the oscillation could be an internal atmospheric instability or a response of the atmosphere to an independent external forcing. Such a forcing could involve changes in solar activity.

Intrigued by these preliminary results, we have undertaken a more comprehensive analysis and applied SSA not only to sunspot number (ISSN) and MJO indices, but also to other solar and climate indices, focusing here on periods longer than annual: Polar Faculae (PF), Pacific Decadal Oscillation (PDO), ENSO, Arctic Oscillation (AAO), Atlantic Multidecadal oscillation (AMO), Tropical Southern Atlantic oscillation (TSA), Western Hemisphere Warm Pool (WHWP), plus the series of Northeast Brazil Rainfall Anomaly and Sahel Standardized Rainfall. We find that the first SSA eigenvectors are in general combinations of rather pure 11-, 5.5-, and 3.6-year pseudo-cycles.

We first give a brief account of SSA, then present the sources of the data, subject them to SSA (starting in more detail with MJO indices), and describe the results. We introduce a first, tentative model of phase evolution of these pseudo-cycles in terms of coupled, nonlinear Kuramoto oscillators. We end with a discussion, in which we evoke mechanisms that could explain why these periodicities are observed in many climate indices. We conclude that there is strong evidence that the variability of the atmosphere contains outstanding components that are forced by solar variability.

\section{SSA}

In this section, we outline the SSA method. We refer the reader to Golyandina et al. (2001) for a full monograph; to Vautard and Ghil (1989), Vautard et al. (1992), and Ghil et al. (2002) for useful accounts; and to Le Mouël et al. (2017) for the way in which we use the method. However, there were some misprints in section 2 of that paper, and we prefer to restate the method with these misprints corrected. SSA is a powerful, nonparametric time series analysis tool that relies on the Karhunen-Loeve spectral decomposition (Kittler \& Young, 1973). Consider the real-valued time series $\boldsymbol{x}=\left\{x_{n}: n=1, \ldots, N\right\}$. SSA decomposes the vector $\boldsymbol{x}$ into a sum of physical components that may be subsequently identified as trends, quasiperiodic oscillations or noise. The starting point is to embed $\boldsymbol{x}$ into an $L$-dimensional vector space, by using lagged copies $\boldsymbol{x}_{L}$ of $\boldsymbol{x}$ which are $K=N-L+1$ elements long. One forms the "trajectory" (Hankel) matrix $\boldsymbol{X}$ :

$$
\mathbf{X}=\left[\begin{array}{llll}
x_{1} & x_{2} & \cdots & x_{K} \\
x_{2} & x_{3} & \cdots & x_{K+1} \\
\vdots & \vdots & \ddots & \vdots \\
x_{L} & x_{L+11} & \cdots & x_{N}
\end{array}\right]
$$

The next step consists in performing the singular value decomposition (Golub \& Kahan, 1965) of the trajectory matrix $\boldsymbol{S}=\boldsymbol{X} \boldsymbol{X}^{T}$. The real eigenvalues $\lambda_{i}\{i=1, \ldots, L\}$ of $\boldsymbol{X} \boldsymbol{X}^{T}$ are ranked in decreasing order of magnitude. The eigenvectors $\boldsymbol{U}_{\boldsymbol{i}}$ of $\boldsymbol{S}\{i=1, \ldots, L\}$ corresponding to these eigenvalues form an orthogonal system. The eigenvectors $\boldsymbol{V}_{\boldsymbol{i}}$ of $\boldsymbol{X}^{T} \boldsymbol{X}$ also form an orthogonal system. The eigen-triplets $\left(\lambda_{i}, \boldsymbol{U}_{\boldsymbol{i}}, \boldsymbol{V}_{\boldsymbol{i}}\right)$ are linked by $\boldsymbol{V}_{\boldsymbol{i}}=\boldsymbol{X}^{T} \boldsymbol{U}_{\boldsymbol{i}} / \sqrt{\lambda_{i}}$. The $\sqrt{\lambda_{i}}$ are called "singular values." The trajectory matrix can be written as a sum of elementary matrices $\left\{\boldsymbol{X}_{i}: i=1, \ldots, L\right\}, \boldsymbol{X}=\boldsymbol{X}_{1}+\ldots+\boldsymbol{X}_{\mathrm{L}}$. The size of a periodic component corresponding to an eigenvector is encoded in the singular value, and its share of the variance in the eigenvalue (as a fraction of their sum). We recall that if a fundamental eigenvector is found, the strongest periodicity may force other 
variations toward higher harmonics. One will therefore exert some caution when trying to interpret harmonics larger than, say, the third harmonic (a value used by many authors from experience; see also Golyandina et al., 2001, p.31).

Note: In Fourier analysis, harmonics of a fundamental period preserve a constant amplitude ratio and phase, without modulation. The orthogonality of eigenvectors imposes that they are sinusoidal. Such is not the case with SSA and we observe modulations and phase drifts of components: these are therefore not strictly "harmonics." The SSA components do not even require oscillatory eigenvectors and often the trend appears as the first component. SSA is a data-adapted empirical method; components, such as 5.5 years, need not be strictly harmonics of 11 years and are not artifacts of the method.

Once the singular value decomposition is made, the last stage consists in the construction of groups of components. Each group is a subset of matrices $\boldsymbol{X}_{i}$. If $I=\left\{i_{1}, \ldots, i_{p}\right\}$ is a group of indices, the corresponding matrix $\boldsymbol{X}_{I}$ is defined as $\boldsymbol{X}_{I}=\boldsymbol{X}_{i 1}+\ldots+\boldsymbol{X}_{i p}$, and the trajectory matrix becomes $\boldsymbol{X}=\boldsymbol{X}_{I 1}+\ldots+\boldsymbol{X}_{I d}$. The choice of the indices in each group is done empirically. As in all empirical procedures, we recognize that this step, and in particular the choice of parameter L, require practical experience with the method. When eigenvalues are arranged in decreasing order of amplitude, the first eigenvalue often correspond to the trend (when there is a significant one) and eigenvalues that occur in pairs with similar values correspond to pseudo-periodic components. Ghil et al. (2002) note that SSA signals can be analyzed further by using other spectral analysis tools. They show that the maximum entropy method works particularly well on signals enhanced by SSA. We find that the classical Fourier method works particularly well on the time series studied in this paper. When the slope of the eigenvalue spectrum flattens, it is assumed that one has reached the noise.

We first apply the method to two solar indices, ISSN and PF, and to the MJO climate index. Then we undertake the same calculations (with shorter developments) on the set of climate indices PDO, ENSO, AAO, AMO, TSA, WHWP, Brazil, and Sahel rainfalls.

\section{Data}

In this section, we outline the data we use. We refer the reader to Blanter et al. (2012) as far as MJO indices are concerned.

\subsection{Solar Indices}

In order to monitor variations in solar activity, we used two classical indices: the international standard sunspot number ISSN (a proxy of the Sun's toroidal magnetic field component, hence of zonal convection) and the number of PF (a proxy of the poloidal magnetic field component, hence of the open field lines and the solar wind; Munoz-Jaramillo et al., 2012). The daily ISSN (1849-2015) is reported by the Solar Influences Data Analysis Centre (http://sidc.oma.be; Figure 1a, where only the data from 1906 to 2006 have been represented, for comparison with PF data). We used monthly PF data from the northern solar hemisphere (Figure 2a) available through http://www.solardynamo.org/data.html (PF data are available from 1906 to 2006 without any gaps; in the southern solar hemisphere, there are gaps, hence we prefer to use the series of northern hemisphere PF).

\subsection{MJO Indices}

The MJO is an intraseasonal wave that originates in the near-equatorial zone of the Pacific hemisphere and forms the dominant mode of tropical atmospheric variability on an intraseasonal time scale (e.g., Gottschalk et al., 2017; C. Zhang, 2005). MJO events consist primarily in large-scale deep convective rainfall anomalies that propagate slowly eastward from the Indian Ocean, through Indonesia, into the western Pacific where they decay around the dateline. They often reappear over the tropical Atlantic and Africa. The MJO has been found to influence temperatures and rainfall well outside the tropics, in North and South America, and up to high latitudes including the Arctic (e.g., Bond \& Vecchi, 2003; Cassou, 2008; Donald et al., 2006; Jones, 2000; Vecchi \& Bond, 2004). It also influences ENSO and the Australian monsoon. MJO is therefore potentially related to climate at the planetary scale (see f.i. papers by Hendon, 1995; Weickmann \& Sardeshmukh, 1994; Wheeler \& Hendon, 2004; Xue et al., 2002; C. Zhang, 2005). The MJO is a naturally occurring component of the coupled ocean-atmosphere system (Gottschalk et al., 2017). Evidence of its diverse impacts on the global climate system entails a detailed investigation of the phenomenon and of its possible forcings. 


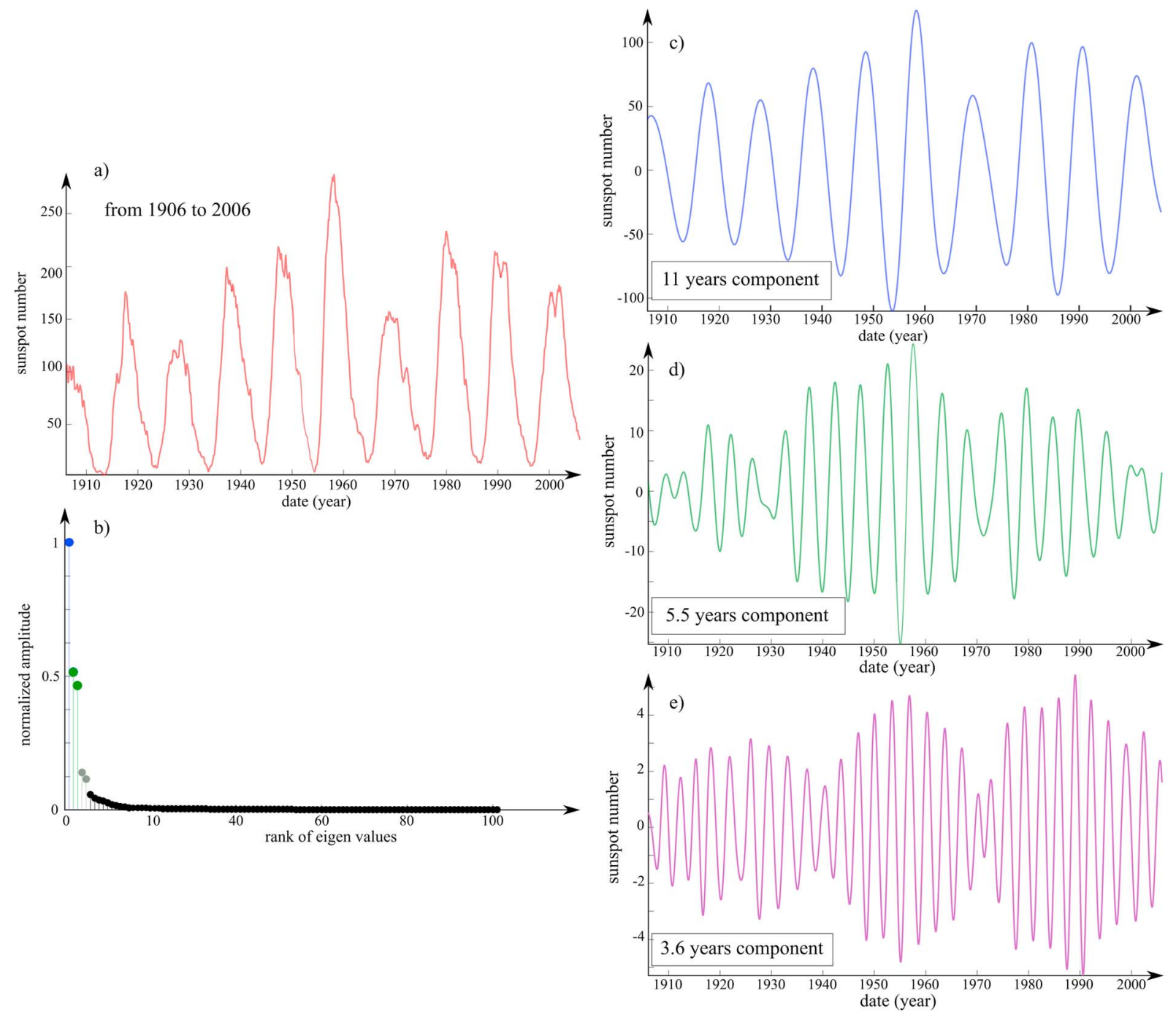

Figure 1. (a) Daily sunspot numbers ISSN 1906-2006; (b) singular spectrum analysis eigenvalues of ISSN time series; (c) first component of singular spectrum analysis (color code same as in Figure 1b)—period 11 years; (d) same as (c) for second component—eigenvalues 2 and 3; period 5.5 years; (e) same as (c) for third component-eigenvalues 4 and 5; period $\sim 3.6$ years.

Daily MJO indices are available through the KNMI access point (http://climexp.knmi.nl/). We consider the set of 10 MJO daily indices (MJO1-MJO10) that are provided by the National Centre for Environmental Prediction, Climate Prediction Centre NCEP/CPC. These indices are built through an Extended Empirical Orthogonal Function (EEOF) analysis from pentad (5 day) 200-hPa velocity potential anomalies equatorward of $30^{\circ} \mathrm{N}$, computed during ENSO-neutral and weak ENSO winters (November-April) in the time interval 4 January 1978 to 24 November 2016. The first EEOF is composed of 10 patterns spaced equally by 5 days from 0 to 45 days (for an illustration of the propagating negative and positive parts of MJO see, e.g., the KNMI access point or Xue et al., 2002, Figure 1). The indices are obtained by regressing the filtered pentad data onto each of the 10 patterns. MJO1-MJO10 are, respectively, relevant to $80^{\circ} \mathrm{E}, 100^{\circ} \mathrm{E}, 120^{\circ} \mathrm{E}, 140^{\circ} \mathrm{E}$, $160^{\circ} \mathrm{E}, 120^{\circ} \mathrm{W}, 40^{\circ} \mathrm{W}, 10^{\circ} \mathrm{W}, 20^{\circ} \mathrm{E}$, and $70^{\circ} \mathrm{E}$ longitudes.

Each index is normalized by its standard deviation. Five out of the 10 MJO indices (MJO10, MJO1, MJO2, MJO3, MJO4) are relevant to the zone of MJO convection (C. Zhang, 2005); each one of the other five indices forms a pair with one of the first five and displays very similar spectral properties (MJO1 and MJO6, MJO2 and $\mathrm{MJO}$, etc. ...). This is a consequence of the symmetry properties of the first EEOF for patterns 25 days apart. MJO10 and MJO1 are relevant to the Indian Ocean, where MJO events usually start, whereas MJO4 is relevant to the western Pacific where the most intense part of the negative MJO pattern ends. The two 


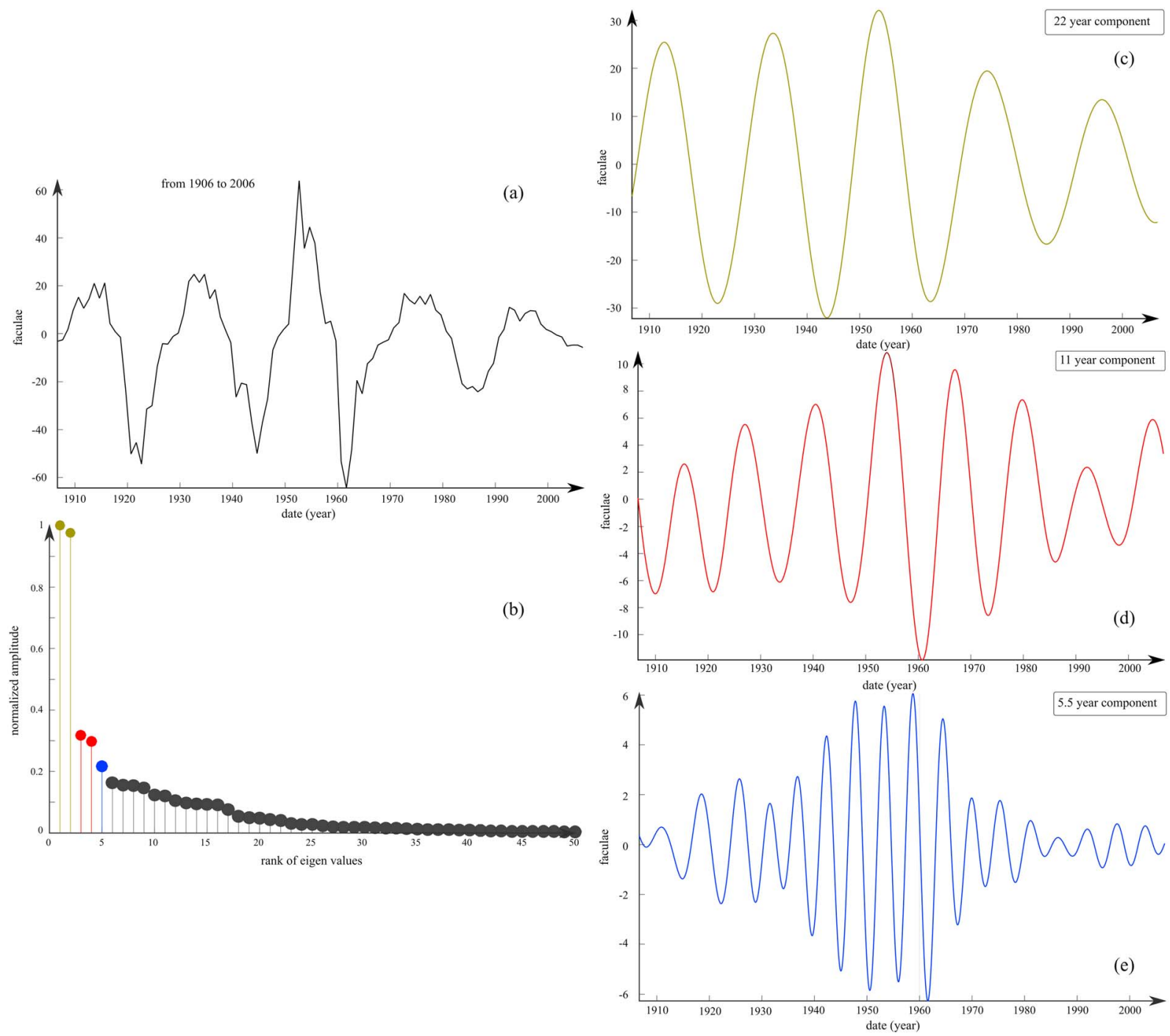

Figure 2. (a) Monthly polar faculae northern solar hemisphere polar faculae 1906-2006; (b) singular spectrum analysis eigenvalues of polar faculae time series; (c) first component of singular spectrum analysis (color code same as in Figure 1b)—eigenvalues 1 and 2; period 22 years; (d) same as (c) for second component—eigenvalues 3 and 4; period 11 years; (e) same as (c) for third component—eigenvalue 5; period 5.5 years.

indices MJO1 and MJO4 and their Fourier spectra are shown in Figure 3 (from Blanter et al., 2012). The smoothed spectra (red curves) actually illustrate the distribution of MJO characteristic (pseudo-) periods in the shorter period domain represented in Figure 3 (20-90 days). The filtering used in the construction of EOFs reduces the contribution of these shorter periods. The main parts of the spectra are concentrated in the 30- to 80-day band, with maximum amplitude in the 40- to 50-day range. There are no simple, sharp periodicities. We have studied this period range using SSA analysis and do find components with sharp periodicities with periods on the order of tens of days but we do not consider these shorter periods further in the present paper. We have investigated in particular the intervals 130-190 days (Rieger oscillations) and 1.2-3.5 years (that includes the quasi-biennial oscillation). We do find (see below, Figure 13) a component with period 3.6 years $(=11 / 3)$. We had identified a component with this period in magnetic observatory data in the late 1970s (Courtillot \& Le Mouël, 1976).

\subsection{Other Climate Indices}

A comprehensive compilation of most climate indices can be found at the site (https://www.esrl.noaa.gov/ $\mathrm{psd} /$ data/climateindices/list/). We have selected the following series: 

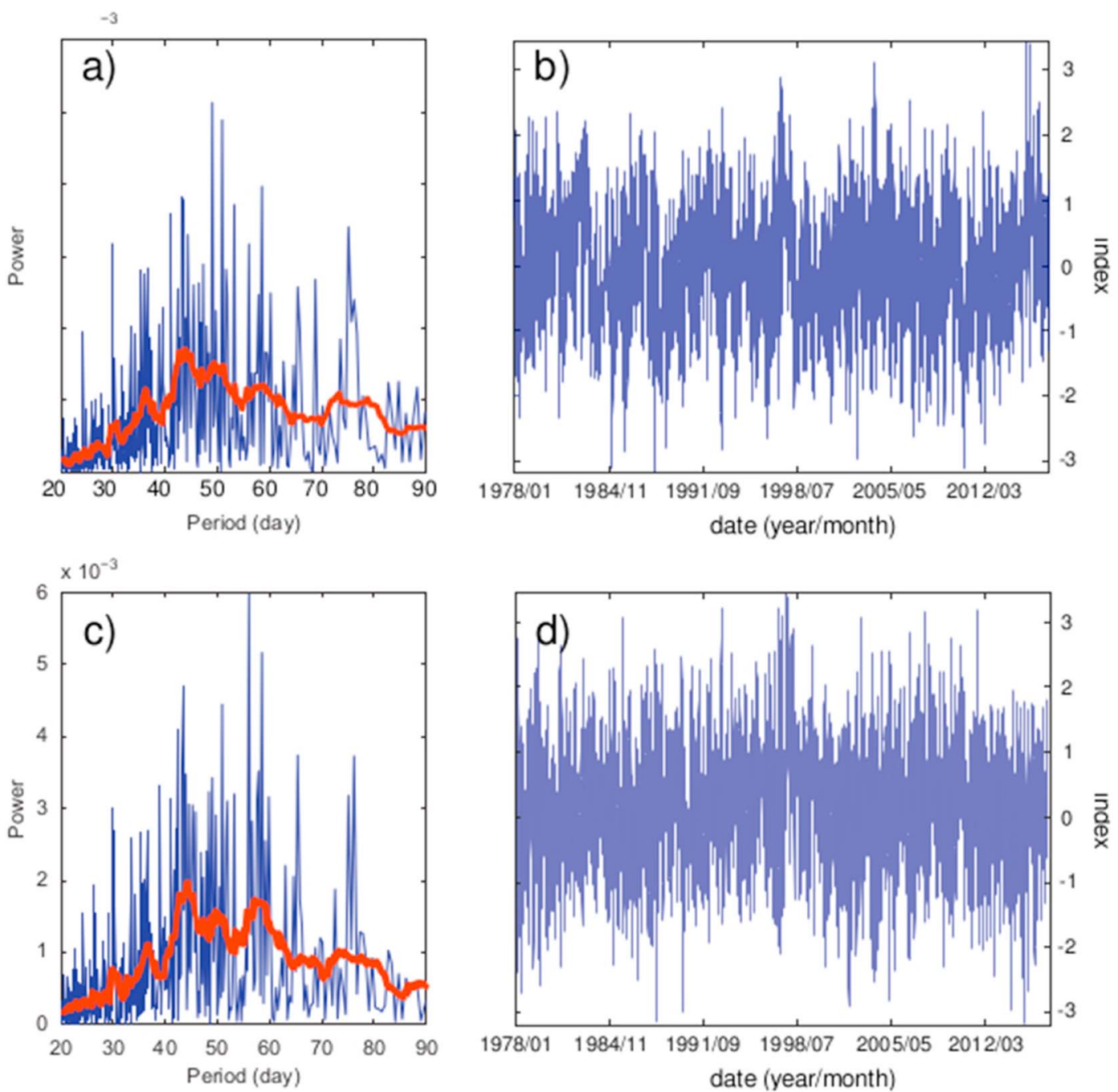

Figure 3. Time series (1978-2016) of the MJO1 (b) and MJO4 (d) Madden-Julian indices and their Fourier power spectra (a and c) (blue) over the 1978-2016 time span with running average taken over 20 frequencies in red (after Blanter et al., 2012).

1. PDO (e.g., Y. Zhang et al., 1997) from October 1974 to April 2017;

2. ENSO (e.g., Curtis \& Adler, 2000) from January 1979 to November 2016;

3. AMO (e.g., Enfield et al., 2001) from January 1948 to March 2017;

4. AAO (see, e.g., Thompson \& Wallace, 1998, 2000) from January 1979 to March 2017;

5. Sahel Standardized Rainfall (20-8 N, 20 W-10E; e.g., Janowiak, 1988) from January 1948 to November 2011;

6. Northeast Brazil Rainfall Anomaly (e.g., Hastenrath \& Greischar, 1993) from January 1948 to December 1999;

7. Tropical Southern Atlantic Index (see, e.g., Enfield et al., 1999) from January 1948 to October 2017;

8. WHWP (see, e.g., Wang \& Enfield, 2001) from January 1948 to October 2017.

We have subjected all these series to SSA.

\section{Results}

We now apply the SSA method, successively, (1) to the ISSN and PF solar indices; (2) to the series of MJO longitudinal indices (with a length $L$ ranging from 4,000 to 11,000 days; see section 2), precisely the daily values from 4 January 1978 to 24 November 2016; and (3) to all eight indices listed in section 3.3. 

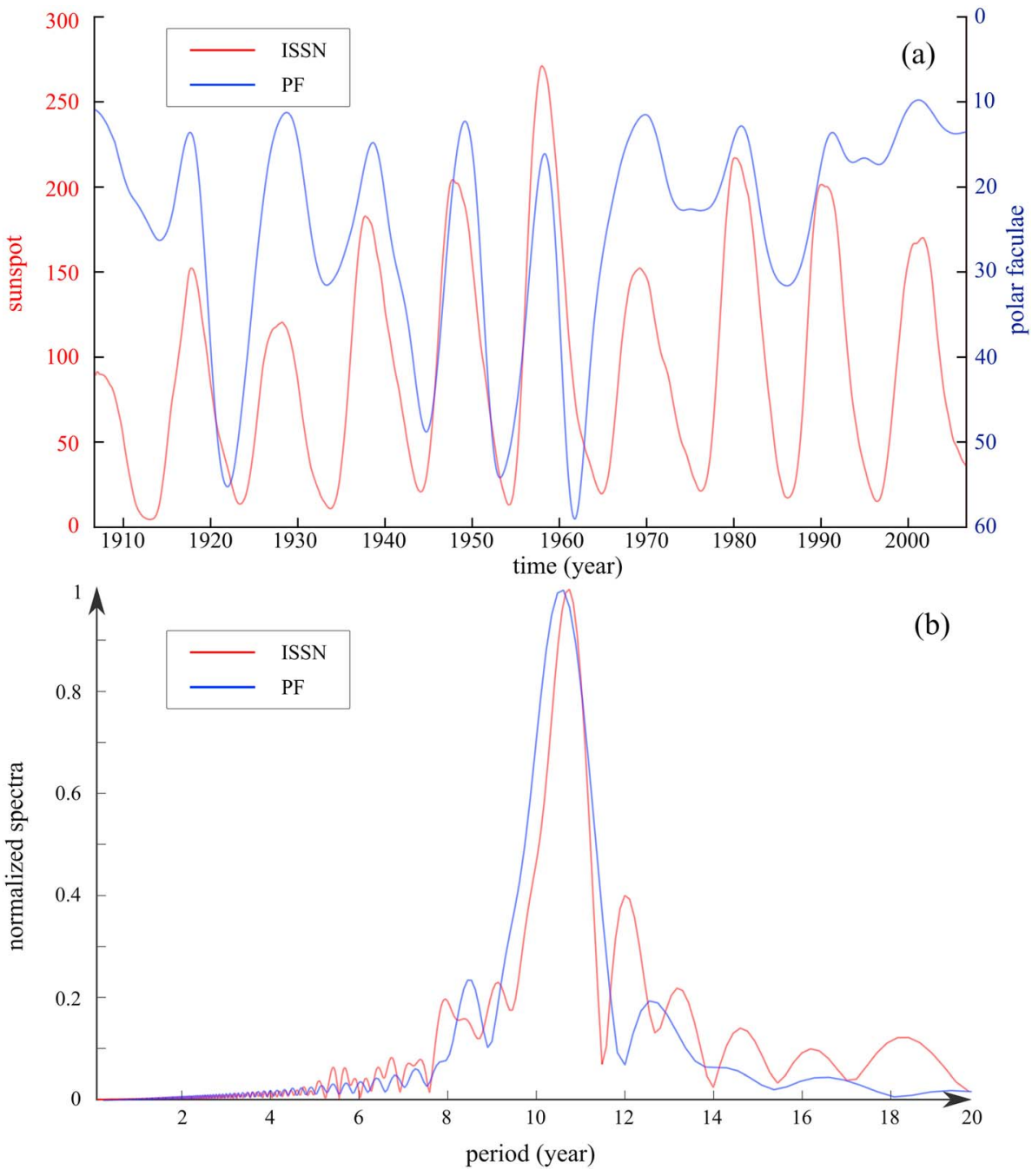

Figure 4. (a) Singular spectrum analysis 11-year ISSN and PF components (note that PF is reversed to display better phase opposition between the two). (b) Fourier spectrum of the two components shown in Figure 4a. ISSN = international sunspot number; $\mathrm{PF}=$ polar faculae.

\subsection{ISSN and PF}

We first analyze solar indices ISSN and PF with SSA. Results are shown in Figures 1 and 2, respectively. Figures $1 \mathrm{~b}$ and $2 \mathrm{~b}$ show the distribution of eigenvalue amplitudes as a function of rank. We see that the first three ISSN SSA components are (in that order) at 11, 5.5, and 3.6 years. The second and third components are, respectively, pairs of the second and third, and fourth and fifth eigenvalues. Taken together these three oscillatory components comprise much of the variance of the total signal, the rest of the eigenvalues corresponding to smaller components and noise (first component $77 \%$ of total variance, second component $15 \%$, and third component 3\%). PF alternates between positive and negative values to account for the Hale magnetic cycle (Makarov et al., 1983; Figure 2a). The first three SSA components of PF are at 22, 11, and 5.5 years, in that order (first component $58 \%$ of total variance, second component $17 \%$, and third component $10 \%$ ). The first pair of eigenvalues (Figure 2b) reflects the 22-year Hale cycle (Figure 2c). The next two SSA components of the decomposition for PF and the first two for ISSN are "11"- and "5.5"-year oscillations (Figures 1b-1d and $2 \mathrm{~d}$ and 2e). The ISSN and PF 11-year SSA components are in phase opposition (Figure 4a); the PF SSA has been reversed to make this visually clear. One can check that the maxima and minima of the ISSN SSA 11-year component coincide closely with the extrema of the original ISSN time series and that the amplitudes follow the same multidecadal trends. Figure $4 \mathrm{~b}$ displays the Fourier spectra of the 11-year SSA components of ISSN and PF in the 1- to 20-year period range. Both spectra display a single strong 

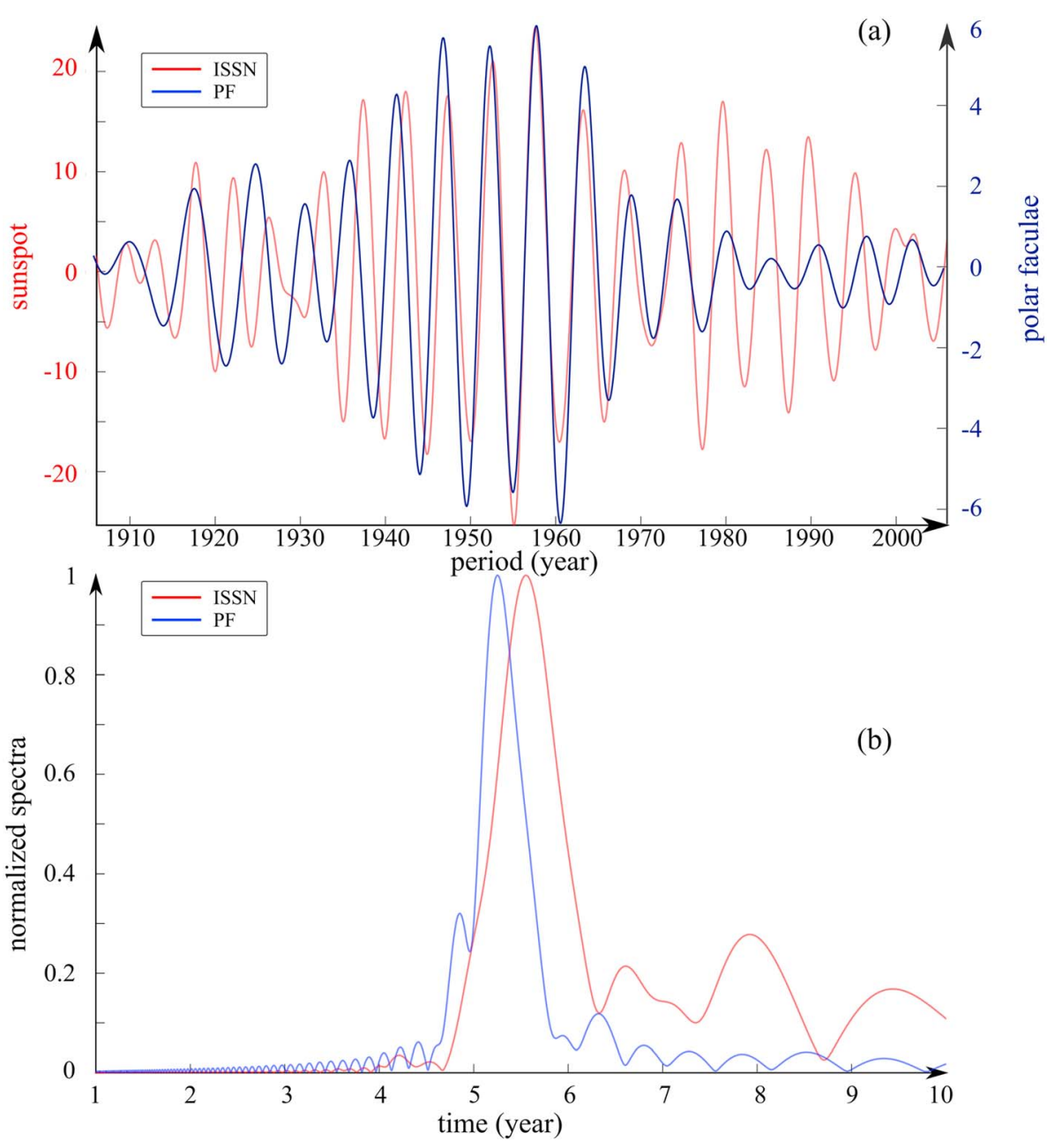

Figure 5. (a) singular spectrum analysis 5.5-year components of ISSN and PF. (b) Fourier spectrum of the two components shown in Figure 5a.

peak at $10.7 \pm 0.6$ and $10.4 \pm 0.8$ year, respectively (for rough estimate of uncertainty, we use the half-width of the peak at mid-height). The ISSN and PF 5.5-year SSA components are shown in Figures 1d and 2e. Both ISSN and PF SSA 5.5-year spectra (Figure 5b) display a single strong peak at $5.6 \pm 0.4$ and $5.3 \pm 0.3$ year, respectively. Figure 1e shows the 3.6-year SSA component of ISSN (see, e.g., Courtillot \& Le Mouël, 1976; Makarov et al., 2001); we found no such component for PF.

\subsection{MJO}

The first 40 eigenvalues of MJO1 are shown in Figure 6 in the case when $L=8,000$ days. The decay of component amplitudes as a function of rank is much slower than that of ISSN in Figure 1b. This reflects the relative importance of both noise and complexity, that are larger in MJO than in ISSN; ISSN can be represented well by a small set of quasiperiodic components, whereas MJO requires a larger set. The eigenvalues in Figure 6 are paired to determine components (section 2). The first component (for the period 1978-2016) is shown in Figure 7 together with its Fourier spectrum. It stands above all others and is remarkably simple: it features a modulated sine wave with a period near 5.5 years. We have checked that this result and those described below are basically unchanged when $L$ ranges from 4,000 to 11,000 days (Figure 8). In some uses of the SSA method (where the focus of the study is on noise reduction and identification of features of chaotic attractors), authors require that window length values $L$ be less than a third of the data length (Ghil et al., 2002; Vautard et al., 1992); here daily data total number $=14205$ and $14205 / 3=4735$. This allows good separation of signal and noise. In order to ensure optimal separation, in the singular value decomposition 


\section{first component}

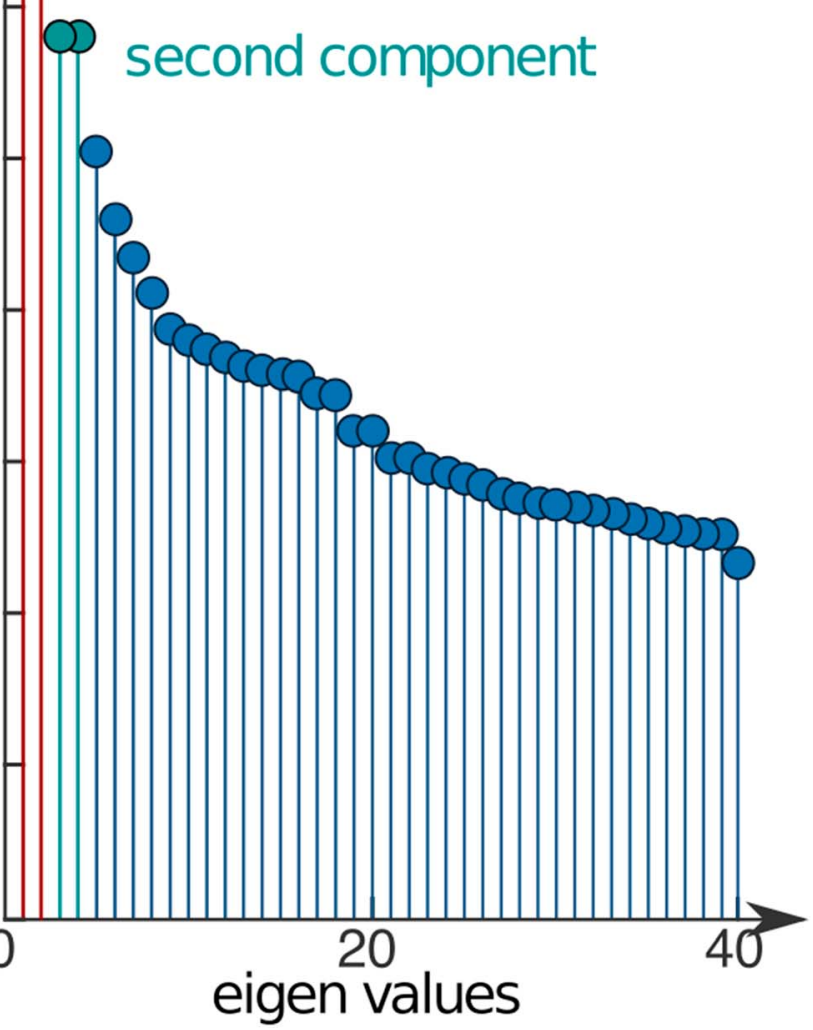

Figure 6. First 100 eigenvalues of the singular spectrum analysis of MJO1 series 1978-2016. of matrix $\boldsymbol{X}$, matrices $\boldsymbol{U}$ and $\boldsymbol{V}$ are made of line and column eigenvectors. In order to get optimal separation, the line and column eigenvectors corresponding to each component should be orthogonal. This is ensured by the algorithm we use (see Golyandina et al., 2001, p. 57; Portes \& Aguirre, 2016). This is confirmed by Figure 8 where the first SSA component for the MJO1 index is almost identical when $\mathrm{L}=8000$ (larger than $14205 / 3$ ) or 4000 (smaller than 14205/3).

The first SSA component for all MJO longitudinal indices is displayed in Figure 9. Seven indices (MJO1, MJO6, MJO2, MJO7, MJO3, MJO5, MJO10) feature the prominent 5.5-year periodicity already seen in Figure 7, whereas the remaining three indices (MJO4, MJO9, MJO8) do not show that period, but instead an 11-year signal. In Figure 9, we have superimposed the ISSN sunspot number series (in red) on the first (5.5or 11-year) SSA component of all MJO indices (in blue). For MJO1, every alternate maximum of the modulated sine component 1 starting with the first maximum correlates with the maximum of the ISSN series, with a phase lag of about 1 year (with ISSN leading). The same is true with every alternate minimum, as is best seen in the other member of the pair, MJO6 (which is in antiphase with MJO1). The amplitude of ISSN decreases over the 1978-2016 period under study and so does that of component 1 of MJO1. It is particularly noteworthy that the "instantaneous" period of component 1 of MJO1 increases in unison with the increase in duration of individual sunspot cycles. Similar observations can be made on component 1 of the pairs MJO2-MJO7 and MJO10-MJO5, that are also (signinverted) images of each other.

The remaining two pairs behave in a different way. The pair MJO4-MJO9 does not display a 5.5-year period first component, but one with a period of 11 years that actually follows the solar cycle. Maxima and minima of that component are well correlated with those of ISSN, again with a slight phase lag of MJO with respect to ISSN (except at the 2009 solar minimum where the small phase difference is in the other sense). Also, the amplitude of the SSA component 1 increases as ISSN cycle amplitude decreases. The remaining pair displays a different, puzzling situation with component 1 of MJO3 displaying the 5.5-year signal and MJO8 the 11-year component. The phase and amplitude characteristics of both are not as clear as for the other four pairs of indices.
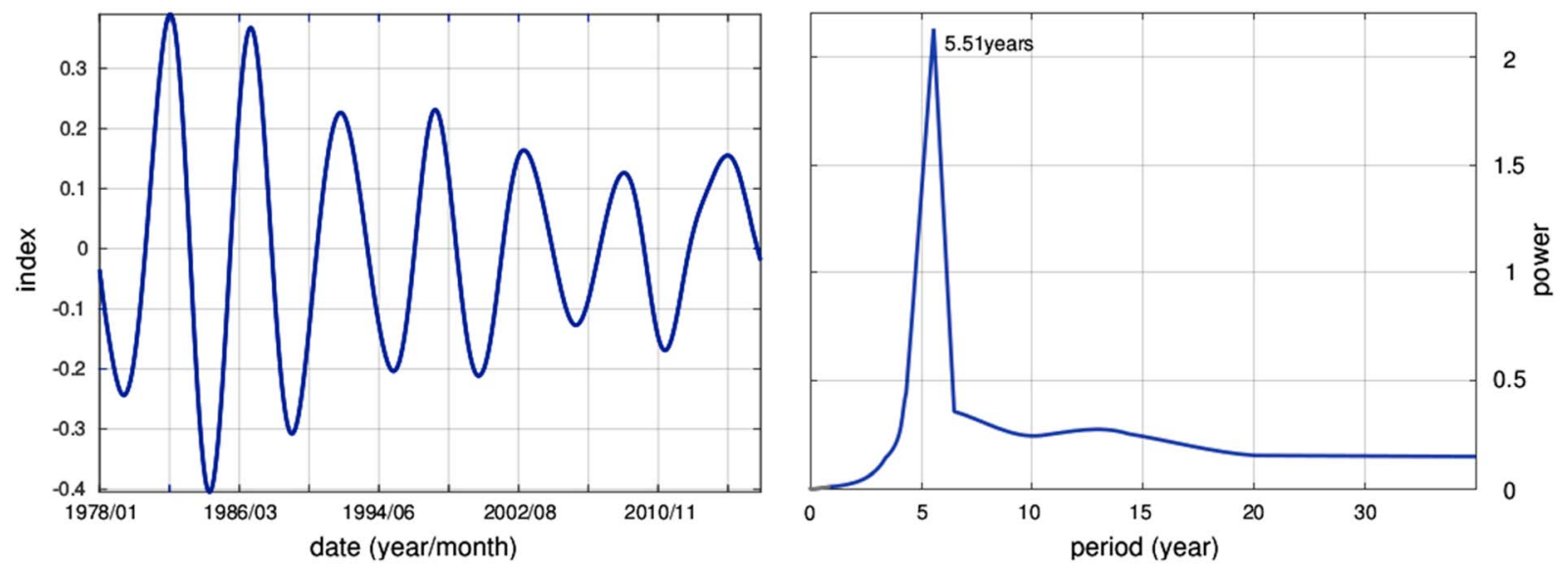

Figure 7. First singular spectrum analysis component of MJO1 index (left column) and its Fourier spectrum (right column). 

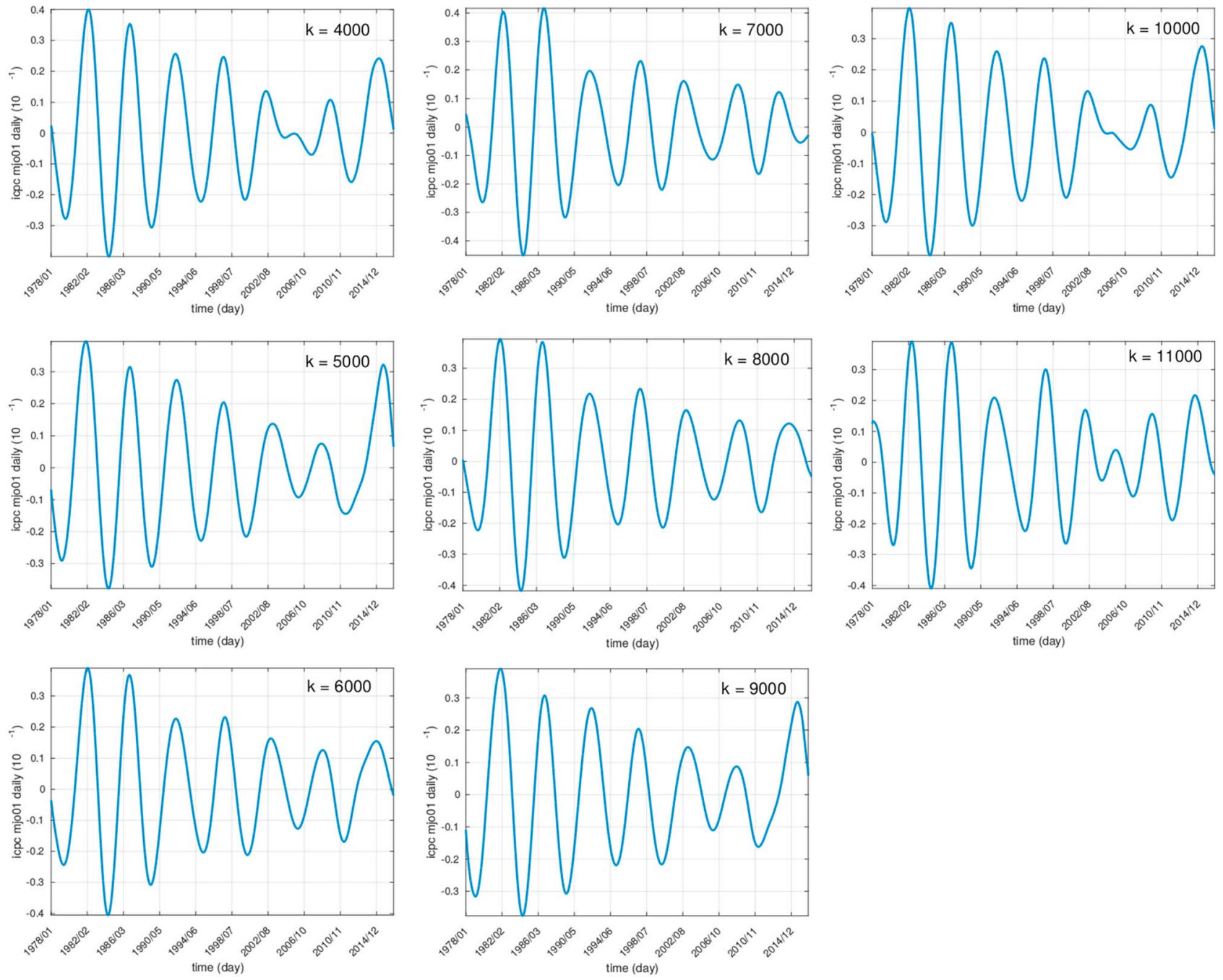

Figure 8. First component of singular spectrum analysis for MJO1 index with L values ranging from 4,000 to 11,000 days (see text).

Figure 10 shows the first, most important SSA component of MJO1 from 1978 to 2016, superimposed on the second component for ISSN (both with periods close to 5.5 years). There is excellent phase agreement from 1980 to 2000, when a phase jump occurs; afterward, the phase difference remains constant up to 2015 (we return to this phase jump in section 5 on Kuramoto modeling). Fourier analysis shows that the main spectral peaks for the two SSA components are at $5.5 \pm 0.7$ and $5.6 \pm 0.6$ year, respectively.

We now seek whether similar observations can be made on other climate indices.

\subsection{PDO, ENSO, AAO, AMO, WHWP, and Brazil and Sahel Rainfalls}

We have subjected all these series to SSA, as done above for ISSN, PF, and MJO. We have found in most cases that among the first to third eigenvalue components, there were eigenvectors with 5.5-, 11-, and also 3.6-year periodicities. Figure 11a shows the "11-year" SSA component for WHWP (component 1-eigenvalues 1 and 2), PDO (component 1-eigenvalues 1 and 2), Sahel rainfall (component 2-eigenvalues 4 and 5; eigenvalues 1 to 3 are at the annual period), and Brazil rainfall (component 2-eigenvalues 4 and 5; eigenvalues 1 to 3 are at the annual period), and MJO and ISSN for comparison (we note that most other climate indices also have an annual component but at higher ranks and lower amplitudes). Figure 11b shows their Fourier transforms: there is a sharp 11-year peak for the ISSN SSA component, with significant power in the period range 9-15 years. All climate indices have a broader spectral peak that overlaps that of ISSN. Peaks of the MJO, Sahel rainfall, and WHWP component 1 are quite close to it. 

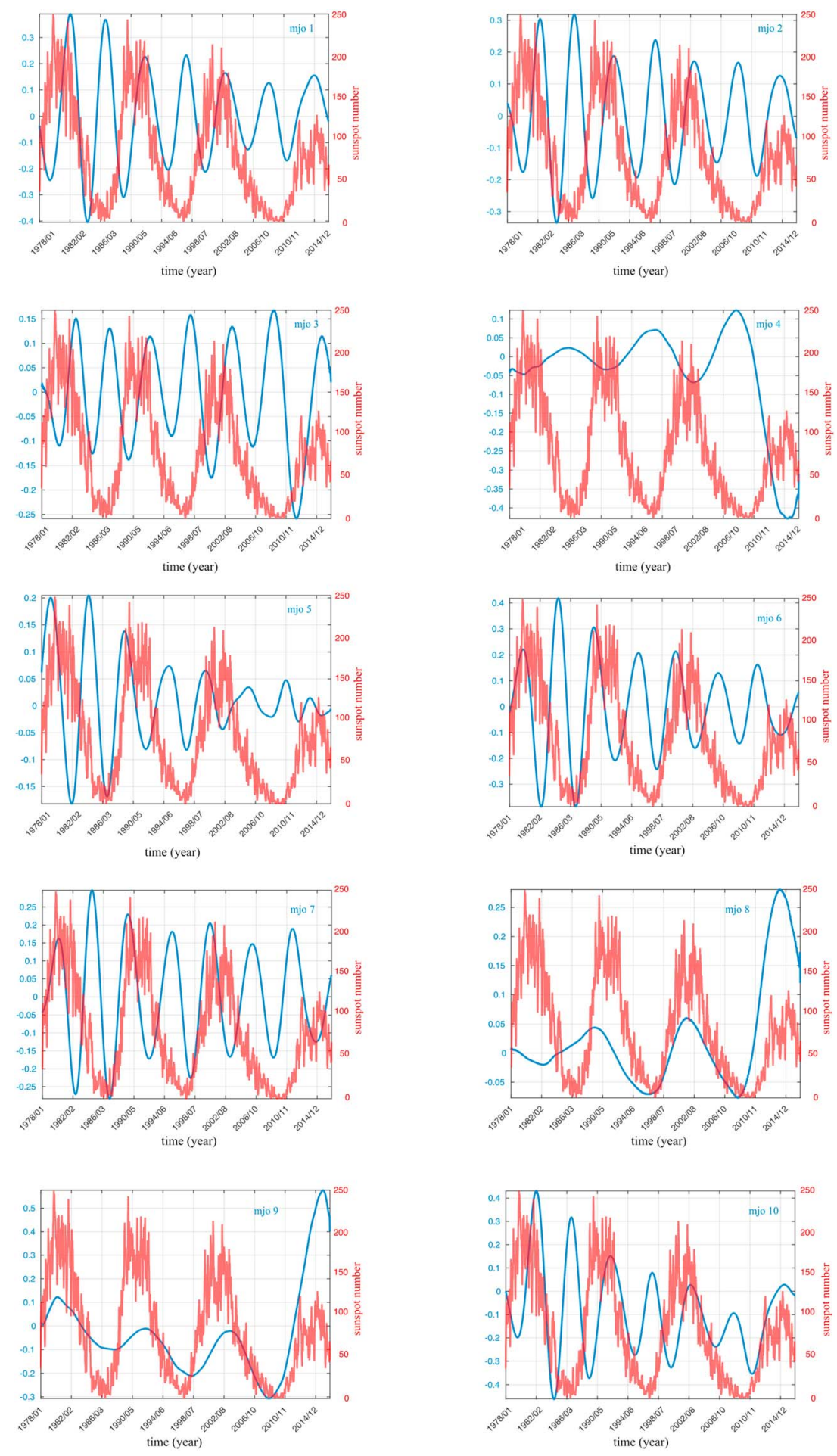

Figure 9. First component of singular spectrum analysis for all $10 \mathrm{MJO}$ indices (in blue) with $\mathrm{L}=8,000$ days. Monthly mean international sunspot number shown in red.

Figure 12a shows the "5.5-year" SSA component for PDO (component 2-eigenvalues 3 and 4), ENSO (component 2-eigenvalue 2), MJO (component 2-eigenvalues 3 and 4), and AAO (component 2-eigenvalue 3), and ISSN for comparison. The corresponding Fourier transforms are shown in Figure 12b. For the 5.5-year SSA components, these peaks occur at periods ranging from 5.3 to 5.6 years, with half-width at 


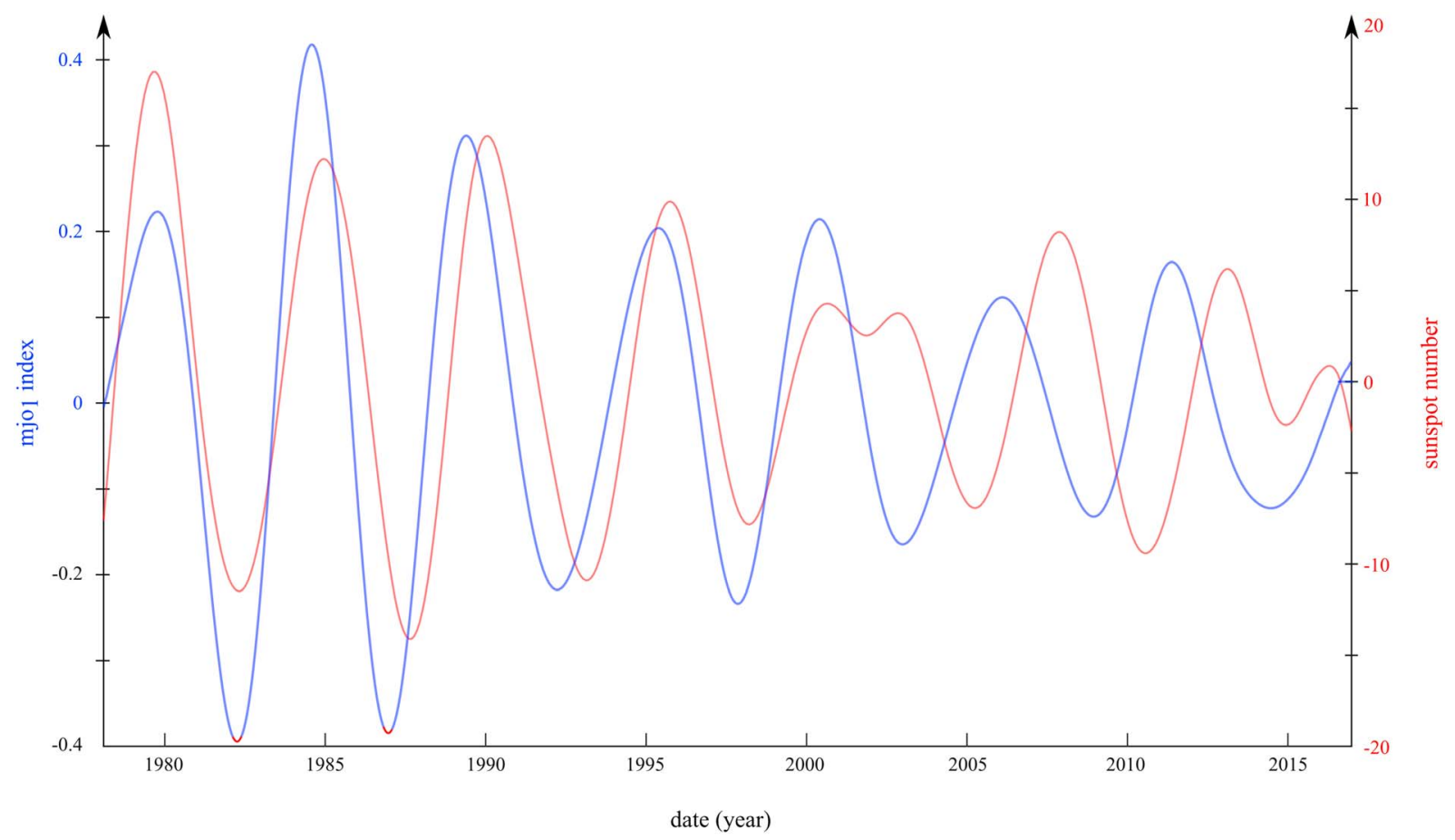

Figure 10. The 5.5-year singular spectrum analysis components of indices international sunspot number and MJO1.

half-peak height ranging from 0.5 to 1.2 years. In other words, should a linear analysis suffice, these peaks could be considered as samples of the same 5.5-year signal with some noise. Comparing Figures 5b and 12b, we see that some of the main components of many major climate indices are found to have a similar importance and period as leading components of a SSA decomposition of solar indices. This is particularly striking for the 5.5-year component (Figure 12b).

\subsection{The 11- and 5.5-Year SSA Components in Climate Indices}

Let us come back in more detail to Figure 11, where we show the 11-year SSA component of climate indices MJO, PDO, WHWP, Sahel, and Brazil rainfall from 1948 to 2017, that we can compare to the corresponding components of the two solar series ISSN and PF (Figure 4b). The 11-year SSA components of the climate indices remain in the same 9-15 years period range (Figure 11a) but drift with respect one to another. In the same time interval, the mean periods of ISSN and PF are, respectively, 10.7 and 10.4 years.

In Figure 10 we have seen that the 5.5-year SSA component of MJO variation is first in phase, then drifts with a phase jump in the early 2000 with respect to that of ISSN. In Figure 12a, we show the 5.5 year SSA components of climate indices PDO, ENSO, AAO, and MJO from 1978 to 2016. MJO is in close phase agreement with ENSO, and also with PDO, which belong to the same large-scale geographical area. Most of them drift with respect to ISSN. AAO is slightly out of phase with respect to ISSN in 1990, then gets in phase at the "2000 phase jump." There are seven oscillations (periods) from 1980 to 2016, corresponding to a mean value of 5.1 years. In the same interval, this number is $5.3 \pm 0.3$ for ISSN and $5.1 \pm 0.3$ for PF.

The most interesting result of the current analysis is shown in Figure 12b: the main spectral peaks of all 5.5year components overlap rather precisely; away from the main peak, on the longer period side, and even on the shorter period side, lobes of the MJO and AAO spectra continue to overlap. Another interesting result is shown in Figure 13 for indices TSA, WHWP, and AMO: there is a clear 3.6-year component in each one of these series, that almost always remains in constant (small) phase difference with respect to the others; Figure 13b shows the same single spectral peak near 3.6 years for all three climate series, all exactly overlapping the main solar spectral peak. The amplitude modulations on the other hand are different, possibly reflecting the different ways in which solar activity forces each (regional) climate subsystem. 


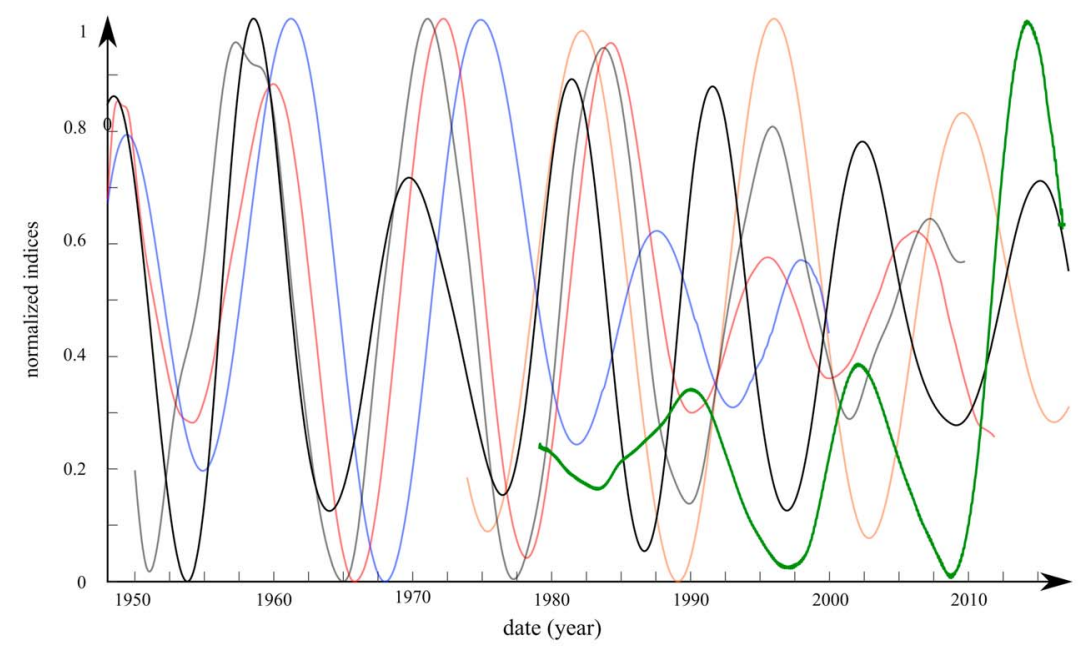

(a)

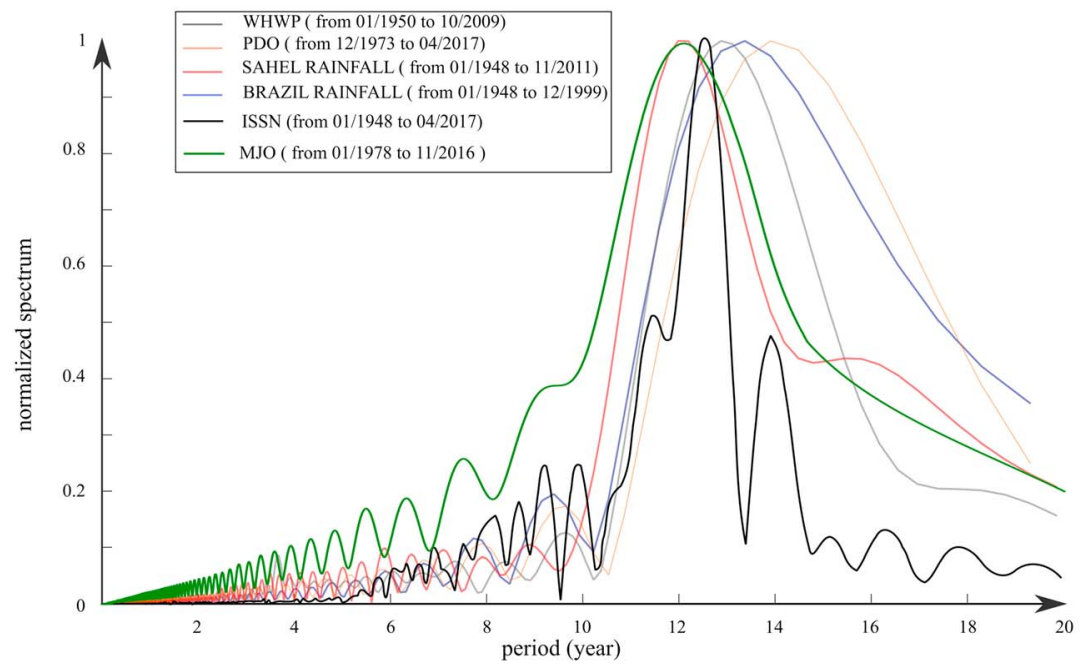

(b)

Figure 11. (a) The 11-year singular spectrum analysis components of indices MJO1, WHWP, PDO, Sahel, Brazil, and ISSN (data dates indicated). (b) Fourier spectra of the components in Figure 11a. WHWP = Western Hemisphere Warm Pool; PDO = Pacific Decadal Oscillation; ISSN = international sunspot number; MJO = Madden-Julian oscillation.

\section{A Kuramoto Model and the Evolution of Phase}

In this section, we attempt to account for some of the observations we are able to make on phases of the SSA components of climate indices, such as the 2000 phase jump seen for instance in Figures 10 and 12. We first note that our data are not easily amenable to an accurate study of amplitudes. Among other reasons, indices are constructed from a mix of variables that do not even have the same physical dimension. Therefore, we rely on phases most of the time, rather than on amplitudes, in the spirit of Kuramoto models (e.g., Acebron et al., 2005; Blanter et al., 2016, 2017).

Blanter et al. $(2016,2017)$ introduced oscillators in order to represent the quasi cyclic behavior of ISSN and $\mathrm{PF}$, respectively, considered as proxies of the equatorial (toroidal) and polar (poloidal) magnetic fields of the Sun. We reconstructed the natural frequencies of these oscillators from the phases $\Phi_{\mathrm{E}}(t)$ and $\Phi_{\mathrm{P}}(t)$ of the corresponding series ISSN and PF with respect to $\sin \Omega \mathrm{t}, \Omega=2 \pi / T, T=10.75$ year (the mean of the period of solar cycles in the interval 1900-2010). We showed that the phases of the oscillations produced by a Kuramoto model corresponding to these natural frequencies were close to the observed phases $\Phi_{\mathrm{E}}(t)$ and $\Phi_{\mathrm{P}}(t)$.

In the present study, we first compute the phases of the 11-year SSA components of both series, that we denote $\Phi_{\mathrm{E}, 11}(t)$ and $\Phi_{\mathrm{P}, 11}(t)$, instead of the phases of ISSN and PF themselves, using the Hilbert transform. 


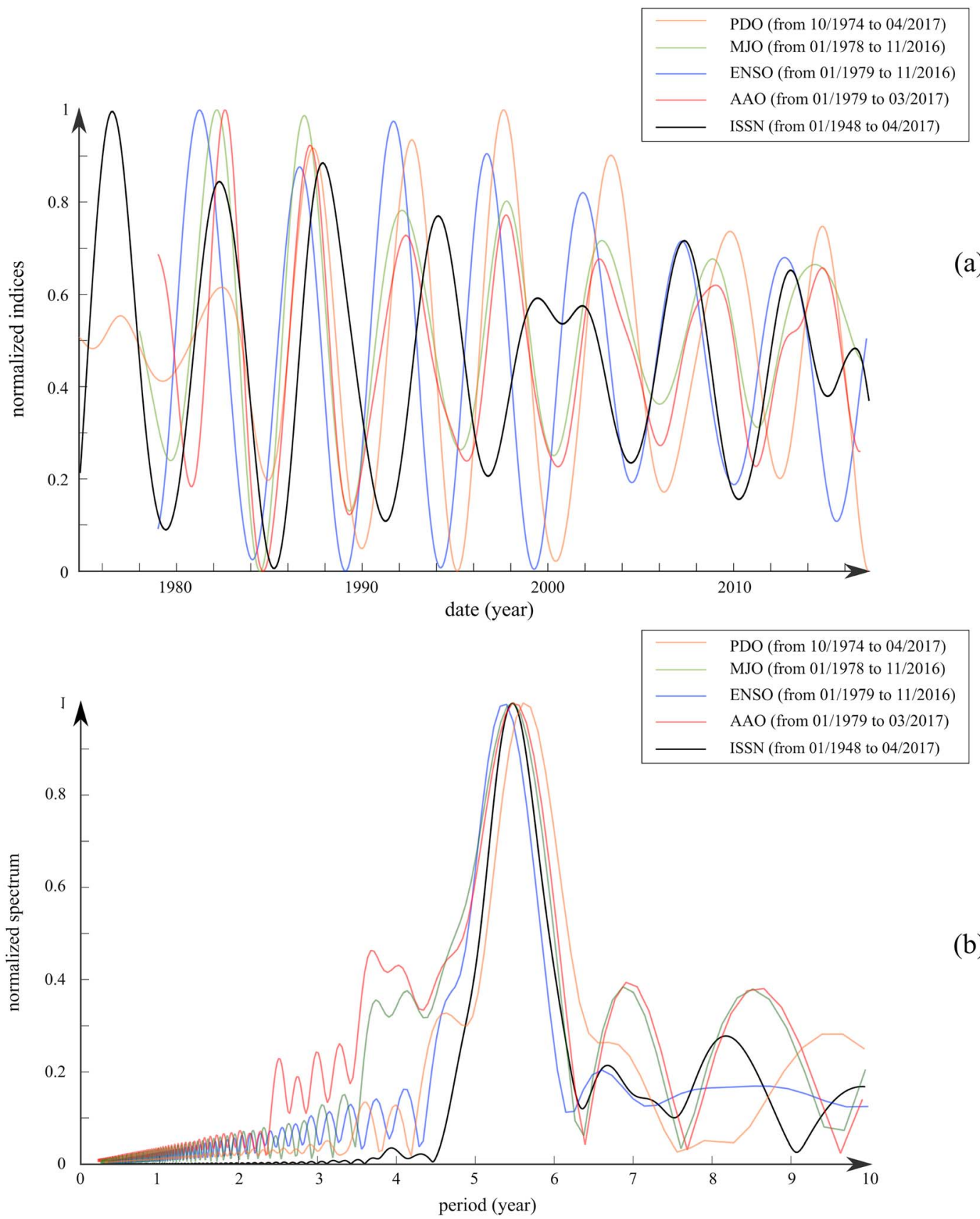

Figure 12. (a) The 5.5-year singular spectrum analysis components of indices PDO, ENSO, AAO, MJO1, and ISSN (data dates indicated). (b) Fourier spectra of the components in Figure 12a. PDO = Pacific Decadal Oscillation; MJO = Madden-Julian oscillation; ENSO = El Nino Southern Oscillation; AAO = Arctic Oscillation; ISSN = international sunspot number.

We next compute in the same way the phases $\Phi_{\mathrm{Ii}, 11}(\mathrm{t})$ of the 11-year components of the different climate indices $I_{\mathrm{i}}(i=1$ to 10$)$, using the same method. We then proceed in the same way for the 5.5year component.

We next represent all series that share common periodicities (11 and 5.5 years, but we could also do it for 3.6 years) by an oscillator. Every terrestrial oscillator $\mathrm{I}_{\mathrm{i}}$ is coupled with both oscillators ISSN and PF. Of course, the reverse does not hold. The presence of approximately the same periods over long time intervals strongly suggests that these oscillators are coupled but not locked (their phase differences can vary). Phase synchronization is well described by Kuramoto models (Acebron et al., 2005). 

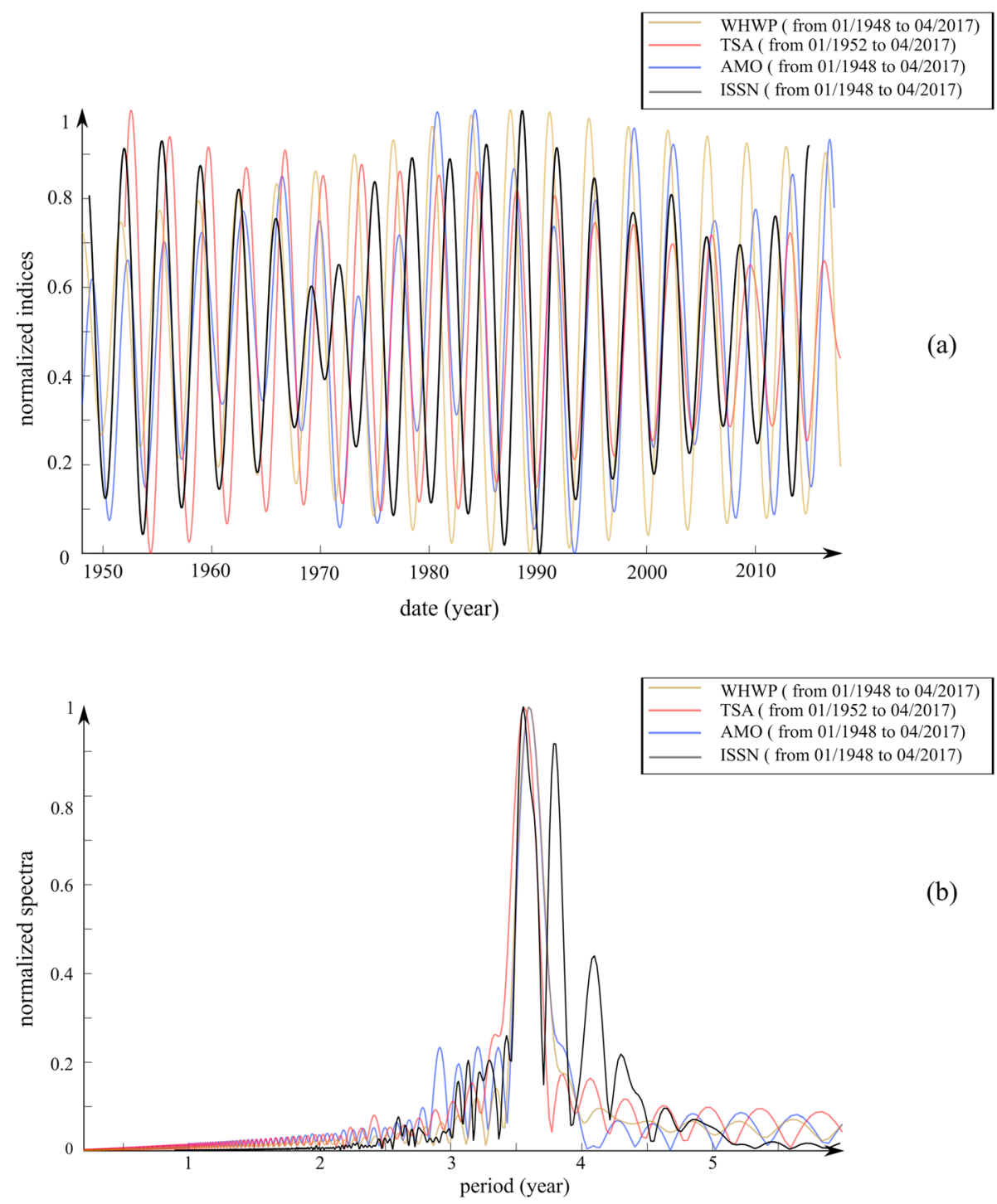

Figure 13. (a) The 3.6-year singular spectrum analysis components of indices TSA, WHWP, AMO, and ISSN (data dates indicated). (b) Fourier spectra of the components in Figure 13a. TSA = Tropical Southern Atlantic oscillation; WHWP = Western Hemisphere Warm Pool; AMO = Atlantic Multidecadal oscillation; ISSN = international sunspot number.

In principle, we consider three oscillators: 1 (sunspots), 2 (faculae), and $3\left(I_{i}, i=1, \ldots, 10\right)$. We have then 10 trios of Kuramoto oscillators. $\theta_{1}(t), \theta_{2}(t)$, and $\theta_{3}(t)$ being the phases of the oscillators, each of the 10 Kuramoto systems can successively be written (the dot is for the time derivative):

$$
\begin{gathered}
\dot{\theta}_{1}=\omega_{1}+k_{12} \sin \left(\theta_{2}-\theta_{1}\right), \\
\dot{\theta}_{2}=\omega_{2}+k_{21} \sin \left(\theta_{1}-\theta_{2}\right), \\
\dot{\theta}_{3}=\omega_{3}+k_{31} \sin \left(\theta_{1}-\theta_{3}\right)+k_{32} \sin \left(\theta_{2}-\theta_{3}\right) .
\end{gathered}
$$

Differential equations (1) and (2) are for the solar oscillators (i.e., sunspots and faculae), (3) for the terrestrial oscillator (i.e., climate index) under consideration; $\omega_{i}(i=1,2$, and 3$)$ are the natural frequencies (all three close to $\Omega=2 \pi / T, T=10.75$ years, in the case of what we call the 11-year variation; the same for the 5.5 year variation, ...). The $k_{\alpha \beta}$ are the coupling coefficients. The $\theta_{i}$ can be computed, given the $\omega_{i}$, the $k_{\alpha \beta}$ and the initial conditions $\theta_{1}^{0}, \theta_{2}^{0}, \theta_{3}^{0}$. 


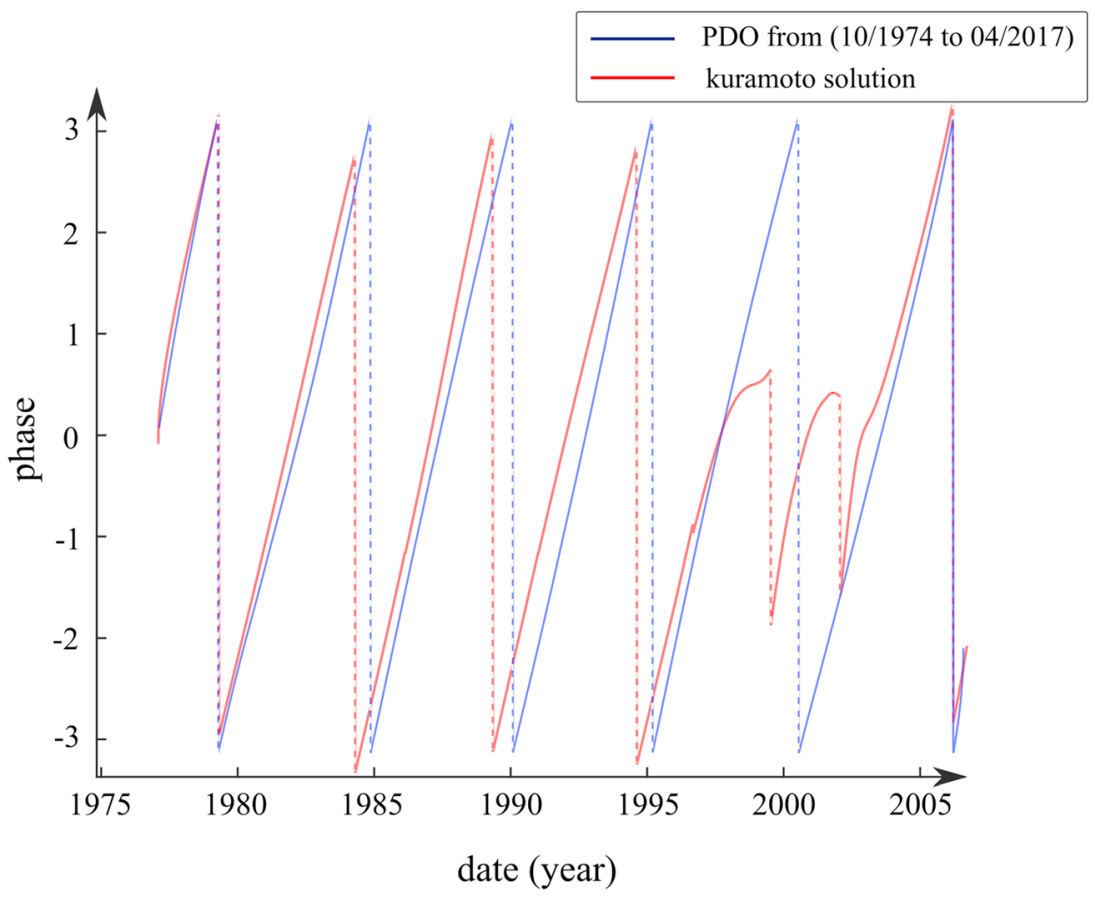

Figure 14. Comparison between the "observed" phase $\widehat{\theta_{\mathrm{PDO}}}=\Omega \mathrm{t}+\widehat{\Phi}_{\mathrm{PDO}}$ of the PDO index (1974-2017) and the computed phase $\theta_{\mathrm{PDO}}=\Omega \mathrm{t}+\Phi_{\mathrm{PDO}}$ of a Kuramoto model of coupled oscillators. The solution depends on the initial condition (time $\mathrm{t}=\mathrm{c}$ ) and the coupling coefficient $\mathrm{k}$. The "best" values of $\mathrm{k}$ and $\mathrm{c}$ are computed using simulated annealing. The Kuramoto phase is computed with $\mathrm{c}=2.3$ years (close to $\pi / 2$ ) and $\mathrm{k}=0.99 /$ year(close to $1 /$ year) and compared to $\theta_{\text {PDO }}$. All phases are displayed modulo $2 \pi$. Note the phase jumps near year 2000 (see Figures 10 and 12 and text for more details). PDO = Pacific Decadal Oscillation.

In this paper, we restrict ourselves to a simpler computation. We evaluate the phases $\Phi_{E, 11}(t)$ and $\Phi_{P, 11}(t)$, or $\Phi_{E, 5.5}(t)$ and $\Phi_{P, 5.5}(t)$ as SSA components of $\Phi_{E}$ and $\Phi_{P}$ as said above. Then, we calculate the phase $\theta_{3}=\Omega t+\Phi_{3}$ for an index $I_{i}$ and a "period" 11 or 5.5 years (this requires equation (3) only). Eventually, we compare the solution $\theta_{3}$ of the system $(1,2,3)$ to the "observed" phase $\widehat{\theta_{3}}$.

Since we are only looking for an order of magnitude, we further simplify the calculation by taking $k_{31}=k_{32}=k, \omega_{3}=\Omega$. Equation (3) becomes

$$
\dot{\Phi}_{3}=2 k \sin \left(\frac{\Phi_{1}+\Phi_{2}}{2}-\Phi_{3}\right) \cos \left(\frac{\Phi_{1}-\Phi_{2}}{2}\right) .
$$

We suppose further that $\Phi_{1}$ and $\Phi_{2}$ are constant. Equation (4) is then a first order differential equation with separable variables, whose solution can be written (with $\Theta=\Phi_{1}-\Phi_{2}$ ),

$$
\Phi_{3}(t)=\left(\frac{\Phi_{1}+\Phi_{2}}{2}\right)-2 \operatorname{arctg}\left(e^{-2 k \cos \left(\frac{\Theta}{2}\right)(t-c)}\right)
$$

The comparison between the observed phase $\widehat{\theta_{3}}=\Omega t+\widehat{\Phi}_{3}$ and the computed phase $\theta_{3}=\Omega t+\Phi_{3}$ is illustrated in the case of the PDO index 5.5-year component (Figure 14). In fact, we have done a little more work: the solution depends on the initial condition, characterized by time $c$. So, we have two parameters that can be adjusted ( $c$ and $k$ ). We have determined the "best" values of $k$ and $c$ using simulated annealing (e.g., Kirkpatrick et al., 1983). The phase $\theta_{P D O}$ illustrated by Figure 14 is computed with $c=2.3$ years (close to $\pi / 2$ ) and $k=0.99 /$ year (close to 1 year) and compared to the calculated value $\widehat{\theta_{P D O}}$. There is a good fit of the Kuramoto solution (in red) to the calculated value derived from observations (in blue) from 1977 to 1997, with a small drift in phase. Then there is a double phase jump shortly before and soon after 2000. The Kuramoto solution is back in phase from 2003 to 2007. We note on Figure 1d 
that the 5.5-year component of ISSN has a double peaked regime change around 2000 (see also Figures 10 and 12).

\section{Discussion and Conclusions}

It does not come as a surprise that SSA finds 11-, 5.5-, and 3.6-year components as the first three eigenvectors of a solar activity proxy such as the international sunspot number ISSN (Figure 1). In the case of the count of PF (i.e., signed), the sequence is 22, 11, and 5.5 years (Figure 2). The modulation of the amplitude of the components is important. In Figure 1, the average value of ISSN is on the order of 100, the amplitude (half of the minimum to maximum of oscillations) of the 11-year component 1 ranges from about 50 to 100 , that of the 5.5-year component 2 from about 5 to 20, that of the 3.6-year component 3 from 1 to 5. So, these modulations range from 2 to 5 for the various components of ISSN. The corresponding range for PF (Figure 2) is from 2 to 6 .

It may come more as a surprise that the 5.5-year oscillation stands out as the main SSA component of most MJO indices. SSA being an objective method with no preliminary assumption, the fact that, for instance, the first, most intense component that it finds in all MJO indices has either a period of 5.5 or 11 years (Figure 9) makes it difficult to escape the hypothesis that there might be a solar forcing component in MJO. Also, the fact that the 5.5-year SSA component 1 of most MJO indices follows the solar cycles with decreasing amplitude in the time period we study adds strength to that association.

In fact, we find that not only the first SSA eigenvalues and eigenvectors of MJO but also those of most other climate indices we have studied (PDO, ENSO, MJO, AAO, AMO, WHWP, and Brazil and Sahel rainfalls; Figure 12) all belong to the set of values (22, 11, 5.5, 3.6 years), that is, (22, 22/2, 22/4, 22/6). All these periods have been attributed for long to solar activity, even the 3.6-year period (Courtillot \& Le Mouël, 1976; Makarov et al., 2001). These considerations should be sufficient, by themselves, to suggest a causal link between solar activity and pseudo-periodicities in most major climate indices. Of course, we acknowledge that correlation does not imply causality; but here, if there is a causal link, it can only be in the direction from the Sun to the Earth's climate, for example, via solar wind, cosmic rays, and/or UV and EUV components of solar irradiance. It would be a most remarkable (and unlikely) coincidence if all the solar and climate proxies we have analyzed had by chance the very same leading components (such as the 5.5-year component). The distribution and geographic extent of the regions concerned by the indices we have considered have of course the (large) scale of the data distribution used to build them. We can say that the effect of solar activity is "worldwide", although a variability of one index from one region to another is observed, for example, in the dominant periods, 11 or 5.5 years, or in the phases of the different components. The fact that a solar signature (SSA components) is found in most climate indices, pertaining to different regions, implies that there is a global effect in this solar-terrestrial relationship; this is an important result of our study.

We should not be surprised that our various curves for the 11- and 5.5-year SSA components may not be perfectly in phase (of course, a drift in phase implies variations in period). Also, the operator that links cause and effect could well be nonlinear. Parameters of the oscillator (f.i. coupling coefficient $k$ in our schematic Kuramoto model) may change from one large region to another. In the same spirit, Tsonis et al. (2007) have constructed a network of climate indices and investigated their collective behavior. This network synchronized several times in the period 1900-2000 and, when the synchronous state was followed by a steady increase in the coupling between the indices, this synchronous state was destroyed and a new climate state set in (see also Courtillot et al., 2013).

Many of the indices rely on the measurements of wind and atmospheric pressure at 200 and $400 \mathrm{hPa}$ (i.e., $\sim 7$ and $11 \mathrm{~km}$ ); so, solar activity affects winds at these altitudes, that is, the winds in the troposphere, and, altogether, the general circulation. Some indices that rely on precipitations, others that rely also on temperature measurements (PDO, ENSO, WHWP, AMO) exhibit the solar activity periods. A solar effect on pressure, temperature, winds, precipitations indeed in summary means a solar forcing of climate altogether. We (and others) have previously observed in various parts of the globe the signature of the solar cycle in temperature measurements: in the United States (Le Mouël et al., 2008), in Europe, and particularly in the Netherlands (Le Mouël et al., 2009), in the U.S. North Pacific (Courtillot et al., 2010), and in the longest temperature series in Europe, in Prague, Bologna, and Uccle (Kossobokov et al., 2010; Le Mouël et al., 2010). 
A number of mechanisms have been suggested to account for evidence of solar forcing of climate. Blanter et al. (2012) find that the correlation between the mean period of the MJO wave packet and solar indices is strongest for UV related indices. This would support the idea of some form of solar forcing through changes in UV and EUV components of solar irradiance, carried by the solar wind (Tinsley, 2008). The correlation of the MJO SSA components with period 5.5 years is as good with PF as it is with ISSN. This means that the solar wind could be an agent of the transfer of energy from the Sun to the MJO oscillation (subsystem) of the oceanic system and its climatic variations. A precise coupling mechanism remains to be found. Other mechanisms have been considered: galactic cosmic rays are strongly modulated by solar activity (Svensmark, 1998; Svensmark et al., 2013). One could be tempted to link the $\sim 1$ year phase lag (Figure 9) with that seen in cosmic rays (e.g., Usoskin et al., 1997). In a recent paper, Svensmark et al. (2017) show both theoretically and experimentally that ions produced by cosmic rays influence aerosol growth to the critical size of cloud condensation nuclei. Both UV and cosmic ray mechanisms (strongly modulated by solar activity) can therefore act on cloud formation mechanisms and extent of cloud cover and thus modify the heat budget of the atmosphere.

In an extensive review of solar influences on climate, Gray et al. (2010) conclude that, based on a number of observations, it is clear that variations in solar UV radiation directly influence stratospheric temperatures, and that the dynamical response to this heating extends the solar influence downward to the lower stratosphere and to the troposphere. They note that "there are many proposed mechanisms for a downward influence from the lower stratosphere into the troposphere (see reviews by Shepherd, 2002; Haynes, 2005).”

In the present paper, the solar frequency SSA components that we have identified in a number of climate indices call for an influence of solar activity on the regime of winds at 7 to $11 \mathrm{~km}$ altitudes, on tropospheric (ground) temperatures and on precipitations. Therefore, the solar effect occurs down to the surface of the solid Earth. Moreover, in some recent papers (Le Mouël et al., 2010; Lopes et al., 2017), we found evidence of a forcing of the solid Earth by solar variability: in Le Mouël et al. (2010), we studied the evolution of the amplitude A of the semiannual variation of the length-of-day (lod) from 1962 to 2009 and showed that it was strongly modulated (up to $30 \%$ ) by the 11-year cycle. Because the semiannual variation of lod and ISSN are anticorrelated, ISSN leading by 1 year, it is correlated with galactic cosmic ray intensity. The main part of the semiannual variation in lod is due to the variation in mean zonal winds. We concluded that variations in mean zonal winds are modulated by the solar activity cycle through variations in irradiance, solar wind, or cosmic ray intensity. In Lopes et al. (2017), we addressed the motion of the pole, that is, the departure of the rotation axis from the polar symmetry axis of the mantle. The components of the rotation in the equatorial plane show clear solar periodicities of 5.5 and 11 years.

We are particularly grateful to an anonymous reviewer who helped us clarify many aspects of the original manuscript and to correct some errors in the description of SSA. The data used in the paper are accessible at the following sites: daily ISSN (1849-2015) from Solar Influences Data Analysis Centre http://sidc.oma.be; monthly polar faculae from northern solar hemisphere (1906-2006 from http://www.solardynamo.org/data. html; and in https://www.esrl.noaa. gov/psd/data/climateindices/list/: Pacific decadal oscillation PDO from October 1974 to April 2017; El Nino Southern oscillation precipitation index ENSO from January 1979 to November 2016; Atlantic multidecadal oscillation AMO from January 1948 to March 2017; Arctic oscillation AAO from January 1979 to March 2017; Sahel Standardized Rainfall (20-8 N, $20 \mathrm{~W}-10^{\circ} \mathrm{E}$ ) from January 1948 to November 2011; Northeast Brazil Rainfall Anomaly from January 1948 to December 1999; Tropical Southern Atlantic Index from January 1948 to October 2017; Western Hemisphere Warm Pool from January 1948 to October 2017. IPGP Contribution 4009.
In conclusion, the analysis of several important climate indices reported in this paper strongly supports the idea that features of regional to global climatic importance are modulated in a very significant way by solar activity variations, far more than the one per mil 11-year variation of total solar irradiance could let one assume. Solar variability impacts many aspects of atmospheric variability on climatic time scales. The solar signature extends from the stratosphere to the solid Earth: the coupling between the troposphere and the solid Earth has been considered for long (e.g., the action of winds on surface topography) and the mechanisms likely involve both zonal and (weaker) vertical exchanges of heat and angular momentum between atmospheric subsystems.

\section{References}

Acebron, J. A., Bonilla, L. L., Perez Vicente, C. J., Ritort, F., \& Spigler, R. (2005). The Kuramoto model: A simple paradigm for synchronization phenomena. Reviews of Modern Physics, 77, 137-185. https://doi.org/10.1103/RevModPhys.77.137

Blanter, E., Le Mouël, J. L., Shnirman, M., \& Courtillot, V. (2012). A correlation of mean period of MJO indices and 11-yr solar variation. Journal of Atmospheric and Solar-Terrestrial Physics, 80, 195-207. https://doi.org/10.1016/j.jastp.2012.01.016

Blanter, E., Le Mouël, J. L., Shnirman, M., \& Courtillot, V. (2017). Reconstruction of the north-south solar asymmetry with a Kuramoto model. Solar Physics, 292(4), 54. https://doi.org/10.1007/s11207-017-1078-3

Blanter, E., Le Mouël, J.-L., Shnirman, M., \& Courtillot, V. (2016). Kuramoto model with non-symmetric coupling reconstructs variations of the solar-cycle period. Solar Physics, 291, 1003-1023. https://doi.org/10.1007/s11207-016-0867-4

Bond, N. A., \& Vecchi, G. A. (2003). The influence of the Madden-Julian oscillation on precipitation in Oregon and Washington. Weather and Forecasting, 18, 600-613. https://doi.org/10.1175/1520-0434(2003)018<0600:TIOTMO >2.0.CO;2

Broomhead, D. S., \& King, G. P. (1986a). Extracting qualitative dynamics from experimental data. Physica D: Nonlinear Phenomena, 20, $217-236$. 
Broomhead, D. S., \& King, G. P. (1986b). On the qualitative analysis of experimental dynamical systems. In S. Sarkar (Ed.), Nonlinear phenomena and chaos (pp. 113-144). Bristol and Boston: Adam Hilger Ltd.

Broomhead, D. S., Jones, R., King, G. P., \& Pike, E. R. (1987). Singular system analysis with application to dynamical systems. In E. R. Pike \& L. A. Lugiato (Eds.), Chaos, noise and fractals (pp. 15-27). Bristol and Boston: Adam Hilger Ltd.

Cassou, C. (2008). Intraseasonal interaction between the Madden-Julian oscillation and the North Atlantic oscillation. Nature, 455, 523-527. https://doi.org/10.1038/nature07286

Courtillot, V., Le Mouël, J. L., Blanter, E., \& Shnirman, M. (2010). Evolution of seasonal temperature disturbances and solar forcing in the US North Pacific. Journal of Atmospheric and Solar - Terrestrial Physics, 72, 83-89. https://doi.org/10.1016/j.jastp.2009.10.011

Courtillot, V., Le Mouël, J. L., Kossobokov, V., Gibert, D., \& Lopes, F. (2013). Multi-decadal trends of global surface temperature: A broken line with alternating 30 yr linear segments? Atmospheric and Climate Sciences, 03, 364-371. https://doi.org/10.4236/ acs.2013.33038

Courtillot, V., \& Le Mouël, J. L. (1976). On the long-period variations of the Earth's magnetic field. Journal of Geophysical Research, 81, 2941-2950. https://doi.org/10.1029/JB081i017p02941

Curtis, S., \& Adler, R. (2000). ENSO indices based on patterns of satellite-derived precipitation. Journal of Climate, 13, 2786-2793. https:// doi.org/10.1175/1520-0442(2000)013<2786:EIBOPO >2.0.CO;2

Donald, A., Meinke, H., Power, B., Wheeler, M. C., Maia A de, H. N., Stone, R. C., et al. (2006). Near-global impact of the Madden-Julian oscillation on rainfall. Geophysical Research Letters, 33, L09704. https://doi.org/10.1029/2005GL025155

Enfield, D. B., Mestas, A. M., Mayer, D. A., \& Cid-Serrano, L. (1999). How ubiquitous is the dipole relationship in tropical Atlantic sea surface temperatures? Journal of Geophysical Research, 104(C4), 7841-7848. https://doi.org/10.1029/1998JC900109

Enfield, D. B., Mestas-Nunez, A. M., \& Trimble, P. J. (2001). The Atlantic multidecadal oscillation and its relation to rainfall and river flows in the continental U.S. Geophysical Research Letters, 28, 2077-2080. https://doi.org/10.1029/2000GL012745

Ghil, M., Allen, M. R., Dettinger, M. D., Ide, K., Kondrashov, D., Mann, M. E., et al. (2002). Advanced spectral methods for climatic time series. Reviews of Geophysics, 40(1), 1003. https://doi.org/10.1029/RG000092

Golub, G., \& Kahan, W. (1965). Calculating the singular values and pseudoinverse of a matrix. Journal of the Society for Industrial and Applied Mathematics - Series B: Numerical Analysis, 2, 205-224. https://doi.org/10.1137/0702016

Golyandina, N., Nekrutkin, V., \& Zhigljasky, A. (2001). Analysis of time series structure: SSA and related techniques, Monographs on Statistics and Applied Probability 90, (p. 295). Boca Raton: Chapman and Hall. https://doi.org/10.1201/9781420035841

Gottschalk, J., Kousky, V., Higgins, W., L'Heureux, M. (2017). Madden-Julian oscillation (MJO) http://www.cpc.ncep.noaa.gov/products/ precip/CWlink/MJO/mjo.shtmlf (downloaded June 20, 2017)

Gray, L. J., Beer, J., Geller, M., Haigh, J. D., Lockwood, M., Matthes, K., et al. (2010). Solar influences on climate. Reviews of Geophysics, 48 , RG4001. https://doi.org/10.1029/2009RG000282

Hastenrath, S., \& Greischar, L. (1993). Circulation mechanisms related to Northeast Brazil rainfall anomalies. Journal of Geophysical Research, 98, 5093-5102. https://doi.org/10.1029/92JD02646

Haynes, P. (2005). Stratospheric dynamics. Annual Review of Fluid Mechanics, 37(1), 263-293. https://doi.org/10.1146/37.061903.175710.

Hendon, H. H. (1995). Length of day changes associated with the Madden-Julian oscillation. Journal of the Atmospheric Sciences, 52, 2373-2383. https://doi.org/10.1175/1520-0469(1995)052<2373:LODCAW >2.0.CO;2

Janowiak, J. E. (1988). An investigation of inter-annual rainfall variability in Africa. Journal of Climate, 1, 240-255. https://doi.org/10.1175/ $1520-0442(1988) 001<0240: \mathrm{AIOIRV}>2.0 . \mathrm{CO} ; 2$

Jones, C. (2000). Occurrence of extreme precipitation events in California and relationships with the Madden-Julian oscillation. Journal of Climate, 13, 3576-3587. https://doi.org/10.1175/1520-0442(2000)013<3576:OOEPEI>2.0.CO;2

Jones, C., \& Carvalho, L. M. V. (2006). Changes in the activity of the Madden-Julian oscillation during 1958-2004. Journal of Climate, 19 , 6353-6370. https://doi.org/10.1175/JCLI3972.1

Kirkpatrick, S., Gelatt, C., Vecchi, M., and others (1983), Optimization by simulated annealing, Science, 220-4598, 671-680.

Kittler, J., \& Young, P. C. (1973). A new approach to feature selection based on the Karhunen-Loeve expansion. Pattern Recognition, 5, 335-352. https://doi.org/10.1016/0031-3203(73)90025-3

Kossobokov, V., Le Mouël, J. L., \& Courtillot, V. (2010). A statistically significant signature of multi-decadal solar activity changes in atmospheric temperatures at three European stations. Journal of Atmospheric and Solar - Terrestrial Physics, 72, 595-606.

Le Mouël, J. L., Blanter, E., Shnirman, M., \& Courtillot, V. (2009). Evidence for solar forcing in variability of temperatures and pressures in Europe. Journal of Atmospheric and Solar - Terrestrial Physics, 71, 1309-1321. https://doi.org/10.1016/j.jastp.2009.05.006

Le Mouël, J.-L., Blanter, E., Shnirman, M., \& Courtillot, V. (2010). Solar forcing of the semi-annual variation of length-of-day. Geophysical Research Letters, 37, L15307. https://doi.org/10.1029/2010GL043185

Le Mouël, J. L., Courtillot, V., Blanter, E., \& Shnirman, M. (2008). Evidence for a solar signature in 20th-century temperature data from the USA and Europe. Comptes Rendus Geoscience, 340, 421-430. https://doi.org/10.1016/j.crte.2008.06.001

Le Mouël, J. L., Lopes, F., \& Courtillot, V. (2017). Identification of Gleissberg cycles and a rising trend in a 315-year-long series of sunspot numbers. Solar Physics, 292, 43. https://doi.org/10.1007/s11207-017-1067-6

Lopes, F., Le Mouël, J. L., \& Gibert, D. (2017). The mantle rotation pole position: A solar component. Comptes Rendus Geoscience, 349, 159-164. https://doi.org/10.1016/j.crte.2017.06.001

Makarov, V. I., Fatianov, M. P., \& Sivaraman, K. R. (1983). Poleward migration of the magnetic neutral line and the reversal of the polar fields on the Sun. Solar Physics, 85(2), 215-226. https://doi.org/10.1007/BF00148649

Makarov, V. I., Tlatov, A. G., \& Sivaraman, K. R. (2001). Does the poleward migration rate of the magnetic fields depend on the strength of the solar cycle? Solar Physics, 202, 11-26. https://doi.org/10.1023/A:1011855103278

Munoz-Jaramillo, A., Sheeley, N., Zhang, J. and de Luca, E. (2012), Calibrating 100 years of polar faculae measurements: Implications for the evolution of the heliospheric magnetic field, The Astrophysical Journal, 753(2), 146. https://doi.org/10.1088/0004-637X/753/ $2 / 146$

Portes, L. L., \& Aguirre, L. A. (2016). Matrix formulation and singular-value decomposition algorithm for structured varimax rotation in multivariate singular spectrum analysis. Physical Review E, 93, 052216.

Shepherd, T. G. (2002). Issues in stratosphere-troposphere coupling. Journal of the Meteorological Society of Japan, 80, 769-792. https://doi. org/10.2151/jmsj.80.769

Shnirman, M. G., Le Mouël, J.-L., \& Blanter, E. M. (2009). The 27-day and 22-year cycles in solar and geomagnetic activity. Solar Physics, 258, 167-179. https://doi.org/10.1007/s11207-009-9395-9

Shnirman, M. G., Le Mouël, J.-L., \& Blanter, E. M. (2010). Slow and fast rotating coronal holes from geomagnetic indices. Solar Physics, 266, 159-171. https://doi.org/10.1007/s11207-010-9605-5 
Svensmark, H. (1998). Influence of cosmic rays on Earth's climate. Physical Review Letters, 81, 5027-5030. https://doi.org/10.1103/ PhysRevLett.81.5027

Svensmark, H., Enghoff, M. B., \& Pedersen, J. O. P. (2013). Response of cloud condensation nuclei ( $>50$ nm) to changes in ion-nucleation Physics Letters A, 377, 2343-2347. https://doi.org/10.1016/j.physleta.2013.07.004

Svensmark, H., Enghoff, M. B., Shaviv, N. J., \& Svensmark, J. (2017). Increased ionization supports growth of aerosols into cloud condensation nuclei. Nature Communications, 8, 2199. https://doi.org/10.1038/s41467-017-02082-2

Thompson, D. W. J., \& Wallace, J. M. (1998). The Arctic oscillation signature in the wintertime geopotential height and temperature fields. Geophysical Research Letters, 25, 1297-1300.

Thompson, D. W. J., \& Wallace, J. M. (2000). Annular modes in the extratropical circulation. Part I: Month-to-month variability. Journal of Climate, 13, 1000-1016. https://doi.org/10.1175/1520-0442(2000)013<1000:AMITEC >2.0.CO;2

Tinsley, B. A. (2008). The global atmospheric electric circuit and its effects on cloud microphysics. Reports on Progress in Physics, 71 , 066801 https://doi.org/10.1088/0034-4885/71/6/066801

Tsonis, A. A., Swanson, K., \& Kravtsov, S. (2007). A new dynamical mechanism for major climate shifts. Geophysical Research Letters, 34, L13705. https://doi.org/10.1029/2007GL030288

Usoskin, I. G., Gallet, Y., Lopes, F., Kovaltsov, G. A., \& Hulot, G. (2016). Solar activity during the Holocene: The Hallstatt cycle and its consequence for grand minima and maxima. Astronomy and Astrophysics, 587, A150. https://doi.org/10.1051/0004-6361/201527295

Usoskin, I. G., Kovaltsov, G. A., Kananen, H., Mursula, K., \& Tanskanen, P. J. (1997). Phase evolution of solar activity and cosmic-ray variation cycles. Solar Physics, 170, 447-452. https://doi.org/10.1023/A:1004971320041

Vautard, R., \& Ghil, M. (1989). Singular spectrum analysis in nonlinear dynamics, with applications to paleoclimatic time series. Physica D: Nonlinear Phenomena, 35, 395-424. https://doi.org/10.1016/0167-2789(89)90077-8

Vautard, R., Yiou, P., \& Ghil, M. (1992). Singular-spectrum analysis: A toolkit for short, noisy chaotic signals. Physica D: Nonlinear Phenomena, 58, 95-126. https://doi.org/10.1016/0167-2789(92)90103-T

Vecchi, G. A., \& Bond, N. A. (2004). The Madden-Julian oscillation (MJO) and northern high latitude wintertime surface air temperatures. Geophysical Research Letters, 31, L04104. https://doi.org/10.1029/2003GL018645

Wang, C., \& Enfield, D. B. (2001). The tropical Western Hemisphere warm pool. Geophysical Research Letters, 28, 1635-1638. https://doi. org/10.1029/2000GL011763

Weickmann, K. M., \& Sardeshmukh, P. D. (1994). The atmospheric angular momentum cycle associated with a Madden-Julian oscillation Journal of the Atmospheric Sciences, 51, 3194-3208. https://doi.org/10.1175/1520-0469(1994)051<3194:TAAMCA>2.0.CO;2

Wheeler, M. C., \& Hendon, H. H. (2004). An all-season real-time multivariate MJO index: Development of an index for monitoring and prediction. Monthly Weather Review, 132, 1917-1932. https://doi.org/10.1175/1520-0493(2004)132<1917:AARMMI>2.0.CO;2

Xue, Y., Higgins, W., \& Kousky, V. (2002). Influences of the Madden-Julian oscillations on temperatures and precipitation in North America during ENSO-neutral and weak ENSO winters. In A workshop on prospects for improved forecasts of weather and short-term climate variability on subseasonal (2 week to 2 month) time scales, (p. 4). Mitchellville, MD: NASA/Godard Space Flight Centre.

Zhang, C. (2005). Madden-Julian oscillation. Reviews of Geophysics, 43, RG2003. https://doi.org/10.1029/2004RG000158

Zhang, Y., Wallace, J. M., \& Battisti, D. S. (1997). ENSO-like interdecadal variability: 1900-93. Journal of Climate, 10, 1004-1020. https:// doi.org/10.1175/1520-0442(1997)010<1004:ELIV>2.0.CO;2 